\title{
Eye Position Effects in Monkey Cortex. I. Visual and Pursuit-Related Activity in Extrastriate Areas MT and MST
}

\author{
F. BREMMER, U. J. ILG, A. THIELE, C. DISTLER, AND K.-P. HOFFMANN \\ Department of Zoology and Neurobiology, Ruhr University Bochum, D-44780 Bochum, Germany
}

\begin{abstract}
Bremmer, F., U. J. Ilg, A. Thiele, C. Distler, and K.-P. Hoffmann. Eye position effects in monkey cortex. I. Visual and pursuitrelated activity in extrastriate areas MT and MST. J. Neurophysiol. 77: 944-961, 1997. We studied the effect of eye position on visual and pursuit-related activity in neurons in the superior temporal sulcus of the macaque monkey. Altogether, 109 neurons from the middle temporal area (area MT) and the medial superior temporal area (area MST) were tested for influence of eye position on their stimulus-driven response in a fixation paradigm. In this paradigm the monitored eye position signal was superimposed onto the stimulus control signal while the monkey fixated at different locations on a screen. This setup guaranteed that an optimized stimulus was moved across the receptive field at the same retinal location for all fixation locations. For $61 \%$ of the MT neurons and $82 \%$ of the MST neurons the stimulus-induced response was modulated by the position of the eyes in the orbit. Directional selectivity was not influenced by eye position. One hundred sixty-eight neurons exhibited direction-specific responses during smooth tracking eye movements and were tested in a pursuit paradigm. Here the monkey had to track a target that started to move in the preferred direction with constant speed from five different locations on the screen in random order. Pursuit-related activity was modulated by eye position in $78 \%$ of the MT neurons as well as in $80 \%$ of the MST neurons tested. Neuronal activity varied linearly as a function of both horizontal and vertical eye position for most of the neurons tested in both areas, i.e., two-dimensional regression planes could be approximated to the responses of most of the neurons. The directions of the gradients of these regression planes correlated neither with the preferred stimulus direction tested in the fixation paradigm nor with the preferred tracking direction in the pursuit paradigm. Eighty-six neurons were tested with both the fixation and the pursuit paradigms. The directions of the gradients of the regression planes fit to the responses in both paradigms tended to correlate with each other, i.e., for more than two thirds of the neurons the angular difference between both directions was less than $\pm 90^{\circ}$. The modulatory effect of the position of the eyes in the orbit proved to balance out at the population level for neurons in areas MT and MST, tested with the fixation as well as the pursuit paradigm. Results are discussed in light of the hypothesis of an ongoing coordinate transformation of the incoming sensory signals into a nonretinocentric representation of the visual field.
\end{abstract}

INTRODUCTION

Visually guided movements are high-order cognitive processes that require transformations between signals used at the input and the output stage. The incoming sensory signals are organized with respect to the retina throughout the visual cortical system and form a retinotopic map within each area (Felleman and van Essen 1991). The outgoing commands from the motor cortical system are organized in spatial or somatotopic maps, i.e., they are related to the external space or single muscles and extremities. The mechanism of the necessary coordinate transformation between the different frames of reference within the visual and the motor system as well as its localization within the cortical system are still unclear. However, two lines of evidence have led to the hypothesis that, at least in primates, this coordinate transformation might be prepared or even accomplished within the posterior parietal cortex (Andersen et al. 1990b).

On the one hand, lesion studies (for reviews see, e.g., Andersen 1987, 1989; Lynch 1980; Stein 1992) in humans as well as nonhuman primates have shown that the posterior parietal cortex plays a crucial role in the processing of visual spatial information. Contrary to lesions within the inferotemporal cortex, which lead to deficits in object recognition and discrimination tasks (Aggleton and Mishkin 1990; Rolls 1991), impairment of the posterior parietal cortex results in deficits in spatial perception and orientation tasks (Stein 1991; Zeki 1988) as well as oculomotor deficits (Braun et al. 1992; Karnath et al. 1991; Lynch 1992; Lynch and McLaren 1979; McLaren and Lynch 1979). These results led Ungerleider and Mishkin (1982) to propose a functional dichotomy within the visual system of humans and nonhuman primates. They suggested two parallel visual streams, both originating from the first visual cortical area, leading either ventrally into the inferotemporal cortex or dorsally into the posterior parietal cortex. It was argued that neurons within the ventral stream might be involved in the processing of object information, whereas neurons within the dorsal stream might be involved in spatial information processing (Goodale and Milner 1992). Single-unit recordings in monkey visual cortex have shown that visual and saccade-related activity of neurons in areas V3A, V6, (Galletti and Battaglini 1989; Galletti et al. 1991, 1995), LIP (lateral intraparietal area), and 7A (Andersen et al. 1985, 1990b) was modulated by the position of the eyes in the orbit. It was suggested (Andersen et al. 1990b, 1993; Galletti et al. 1991; Mazzoni et al. 1991) that these eye-position-dependent neurons might be involved in the construction of a nonretinocentric internal map of the external world. This hypothesis was buttressed by findings of a number of theoretical studies (Andersen and Zipser 1988; Goodman and Andersen 1989, 1990; Pouget et al. 1993; Zipser and Andersen 1988) that revealed that the response characteristics of the recorded LIP and 7A neurons were similar to the response properties of hidden layer neurons of a back-propagation network trained to generate an egocentric representation of space.

Cortical areas V3A, V6, LIP, and 7A are by anatomic definition part of the dorsal pathway of the visual system. However, there are several more interconnected areas constituting this dorsal stream (Andersen et al. 1990a; Boussaoud et al. 1990; Felleman and van Essen 1991; Goodale and 
Milner 1992; Morel and Bullier 1990) not yet tested for a likely existing influence of the position of the eyes in the orbit on their stimulus-induced or oculomotor-related responses. Neurons in the middle temporal area (area MT) and the medial superior temporal area (area MST) play a potential role in the processing of spatial information and therefore in the control of visually guided movement. Because of their functional response characteristics, they are likely to be involved in perception of the direction of heading from optic flow (Duffy and Wurtz 1991a,b, 1995; Lappe and Rauschecker 1993, 1994; Lappe et al. 1994), detection of object motion (Tanaka et al. 1993), control of smooth pursuit eye movements (Komatsu and Wurtz 1988), and control of optokinetic nystagmus (Ilg and Hoffmann 1993). In our study we therefore asked whether neuronal activity in motion-sensitive (dorsal pathway) areas MT and MST is modulated by eye position, too.

Neurons were tested with two different paradigms: the fixation and the pursuit paradigms. For most of the neurons studied, discharge indeed was modulated by eye position. This was true for purely visual responses when the monkey fixated a spot of light during movement of an optimized stimulus across the receptive field (RF) as well as for the pursuit-related responses. For most of the affected neurons the modulatory effect varied linearly with horizontal and vertical eye position. The strength of the modulatory effect was comparable with those described for neurons in areas V3A, V6, LIP, and 7A. It is suggested that the observed modulatory effect of eye position on neuronal responses is a quite common phenomenon within the visual cortical system and might be used for the generation of a representation of the visual environment in a nonretinocentric frame of reference.

\section{METHODS}

Single-cell recordings were performed in awake, behaving monkeys performing fixation and pursuit tasks. Recordings were made from three hemispheres of three monkeys [2 male monkeys ( $\mathrm{Ma}$ caca fascicularis, $6.2 \mathrm{~kg}$; Macaca mulatta, $11.8 \mathrm{~kg}$ ); 1 female monkey (Macaca mulatta, $4.6 \mathrm{~kg}$ )]. All procedures were in accordance with published guidelines on the use of animals in research (European Communities Council Directive 86/609/ECC).

\section{Animal preparation}

All monkeys were surgically prepared for chronic neurophysiological recordings. Monkeys were pretreated with atropine and sedated with ketamine hydrochloride. Under general anesthesia [pentobarbital sodium (Nembutal), $10 \mathrm{mg} / \mathrm{kg}$ iv ] and sterile surgical conditions each animal was implanted with a device for holding the head. Two scleral search coils were implanted to monitor eye position according to the method published by Judge et al. (1980) and were connected to a plug on top of the skull. A recording chamber (plus a 2 nd chamber in the 3rd, female monkey) for introducing a guide tube with an electrode through the intact dura was implanted over a craniotomy. The chamber was placed over occipital cortex in a parasagittal stereotaxic plane tilted $60^{\circ}$ back from the vertical. Recording chamber, eye coil plug, and head holder were all embedded in dental acrylic that itself was connected to the skull by self-tapping screws. Analgesics were applied postoperatively and recording started no sooner than $1 \mathrm{wk}$ after surgery.

\section{Behavioral paradigm and recordings}

During training and recording sessions the monkey was restrained in a primate chair (with the head fixed only during re- cordings) while performing fixation or pursuit tasks for liquid reward (apple juice). Rewards were given for keeping the eyes within an electronically defined window centered on the fixation or pursuit target. Behavioral paradigms, visual stimulation, and data acquisition were controlled by a PC (COMPAQ 386-25) that was run by the software package DADA (Data Aquisition and Data Analysis, U. J. Ilg). At the end of training or experimental sessions the monkey was returned to its cage. The monkey's weight was monitored daily and supplementary fruit or water were provided if necessary.

For cell recordings tungsten-in-glass electrodes (impedance 1$2 \mathrm{M} \Omega$ at $1 \mathrm{kHz})$ were advanced with the use of a hydraulic microdrive (Narishige) that was mounted on the recording chamber. Neuronal activity and electrode depth were noted to establish the relative position of landmarks such as gray and white matter and neuronal response characteristics. During recordings, areas MT and MST were identified by their location within the superior temporal sulcus (STS) as well as by their typical response and RF characteristics (Celebrini and Newsome 1994).

\section{$R F$ mapping and visual stimulation}

When an action potential of a cell was isolated, the RF was mapped with the use of a hand-held projector while the monkey fixated a central target. The target $\left(1.0^{\circ}\right.$ diam, $\left.0.4 \mathrm{~cd} / \mathrm{m}^{2}\right)$ was generated by a light-emitting diode back-projected on a translucent tangent screen subtending $90^{\circ} \times 90^{\circ}$ at a viewing distance of 35 $\mathrm{cm}$. Some MST neurons revealing an RF at least as large as the tangent screen during central fixation were tested for the extent of the RF in the periphery while the monkey fixated different noncentral locations. Background illumination was switched on between recordings to avoid dark adaptation. During recordings the background light was switched off. Thus the monkey, separated from the experimental apparatus by thick black curtains, was in total darkness except for the fixation target and the visual stimulus in the fixation paradigm or the pursuit target in the pursuit paradigm (background luminance $<0.0001 \mathrm{~cd} / \mathrm{m}^{2}$ ), respectively.

Quantitative testing of RF properties was performed with the use of a galvanometer-mounted slide projecting system allowing display of different visual stimuli (light bars or random dot patterns of different size). Bar size ranged from 1 to $20^{\circ}$ (length) and 0.5 to $3^{\circ}$ (width). Random dot patterns were of different size $\left(10^{\circ} \times\right.$ $10^{\circ}, 20^{\circ} \times 20^{\circ}, 30^{\circ} \times 30^{\circ}$, and full field completely covering the tangent screen), consisting of uniformly distributed bright pixels of $2^{\circ} \times 2^{\circ}$ size. Luminance of both light bars and bright pixels of the Julesz pattern was $1.0 \mathrm{~cd} / \mathrm{m}^{2}$. Different stimuli were moved at different velocities across the cell's RF so as to determine the optimal speed and preferred direction of the optimal visual stimulus (oriented light bar or random dot pattern). Directional selectivity of many neurons was assessed by moving a random dot pattern along a circular pathway (continuous mapping of directional selectivity) (see, e.g., Hoffmann and Distler 1989; Schoppmann and Hoffmann 1976). In this paradigm the speed of the stimulus (random dot pattern) is kept constant throughout a stimulus trial (cycle), but stimulus direction is changed continuously $\left(0-360^{\circ}\right)$ within a complete stimulus cycle. Thus each pattern element moves with the same speed (typically 15.7 or $31.4^{\circ} / \mathrm{s}$ ) around its own center of motion (radius typically 5 or $10^{\circ}$ ). This kind of stimulation is very different from typical clockwise or counterclockwise rotation stimuli often used in optic flow experiments, where stimulus elements have only one common center of rotation and increase in speed with increasing eccentricity. The continuous mapping of directional selectivity has two experimental advantages. First, the full two-dimensional stimulus space can be covered during a single trial without the need to test a critical number of unidirectional pattern movements. It has been shown before that the directional tuning obtained with the use of the continuous mapping procedure does not differ from the tuning obtained by unidirectional pattern 
movements (e.g., Hoffmann and Distler 1989; Schoppmann and Hoffmann 1976). Second, the neuronal response can mathematically be treated as a continuous periodic function and thus examined by means of SDO analysis. This mathematical tool, based on Fourier theory (Bettges 1992; Wörgötter and Eysel 1987), is dedicated for computing directional selectivity. In this mathematical approach, the neuronal response [peristimulus time histogram (PSTH)] is approximated by the Fourier retransform

$$
R(t)=a_{0}+\left(a_{1} \cos t+b_{1} \sin t\right)+\left(a_{2} \cos 2 t+b_{2} \sin 2 t\right)
$$

This sum contains terms with order (periodicity) 0,1 , and 2 . By definition, direction of stimulus motion has a periodicity of 1 $\left(360^{\circ}\right)$, whereas orientation is repeated every $180^{\circ}$ (periodicity 2 ). Accordingly, direction $(D)$ is regarded as first-order component and orientation $(O)$ as second-order component of the Fourier retransform $R(t)$ of the neuronal response, whereas mean stimulusinduced activity $(S)$ averaged across one full stimulus cycle is regarded as the zero-order component. The preferred stimulus direction $\left(D_{\mathrm{StimPD}}\right)$ is mathematically given by

$$
S_{\mathrm{StimPD}}=\arctan \left(b_{1} / a_{1}\right)
$$

\section{Fixation paradigm}

To test for the eye position effect, fixation targets were presented in random order at nine different locations on the screen. While the monkey fixated the target an optimized stimulus was moved (wholesale motion) across the RF. During fixation, the monitored eye position signal was superimposed onto the signal driving the stimulus so as to guarantee stimulation of always the same part of the retina (retinal clamp). The recorded eye position signal was linked directly to the stimulus control system without any digitizing or computational steps in between. Thus the superposition of both signals occurred without temporal delay and spatial threshold. The stimulus control system itself was damped at $f=50 \mathrm{~Hz}$, preventing fading of the visual stimulus (Collewijn and Tamminga 1986). An eventually occurring small correctional eye movement within the fixation window was thus compensated for in $<5 \mathrm{~ms}$. Fixation locations were the center of the screen $\left([X, Y]=\left[0^{\circ}, 0^{\circ}\right]\right)$ plus eight concentrically located points usually $15^{\circ}\left([X, Y]=\left[ \pm 15^{\circ}, 0^{\circ}\right]\right.$, $\left.\left[0^{\circ}, \pm 15^{\circ}\right],\left[ \pm 10.6^{\circ}, \pm 10.6^{\circ}\right]\right)$ away from the center.

Neurons were tested with an optimized stimulus moving into preferred and nonpreferred direction. If not noted otherwise, the mean response for the entire stimulus movement into the neuron's preferred direction was used as a measure of neuronal responsiveness at a specific fixation location.

\section{Pursuit paradigm}

Cells were also tested for pursuit-related activity. In a first step, this was done with the step ramp paradigm (Rashbass 1961). In this paradigm, after an initial phase of fixation $(1,000 \mathrm{~ms})$ the central fixation light was extinguished, the pursuit target was switched on in the periphery and moved centripetally with a speed of $15^{\circ} / \mathrm{s}$. Starting points for pursuit were $15^{\circ}$ away from the center and located on both the horizontal and vertical meridians $\left([X, Y]=\left[ \pm 15^{\circ}, 0^{\circ}\right],\left[0^{\circ}, \pm 15^{\circ}\right]\right)$. The result of this testing determined approximately the preferred direction of pursuit of each individual neuron.

To test for an eye position effect on the pursuit-related response, the pursuit paradigm was employed. In this task the monkey, after fixating a central spot of light for $1,000 \mathrm{~ms}$, had to pursue a target that started moving in random order from one of five different locations, always with the same speed into the preferred direction. Starting locations for pursuit were $15^{\circ}$ apart from the center and the center itself $\left([X, Y]=\left[ \pm 10.6^{\circ}, \pm 10.6^{\circ}\right],\left[0^{\circ}, 0^{\circ}\right]\right)$. Target movement lasted for $1,200 \mathrm{~ms}$. As in the fixation paradigm, the electronically defined window had a size of $\pm 2.5^{\circ}$ that enforced the monkeys to perform accurate tracking eye movements.

For analyzing neuronal activity in the pursuit paradigm, individual trials were aligned to the onset of the initial saccade preceding the smooth pursuit phase. Trials typically were divided into two characteristic epochs. Epoch 1 was defined as the time window beginning with trial onset (fixation light on) until $100 \mathrm{~ms}$ before the initial saccade. Activity during this epoch was taken into account as background activity. Epoch 2 was defined as beginning $100 \mathrm{~ms}$ after saccade onset until the end of pursuit target movement. Epoch 2 normally lasted for $\sim 1,000 \mathrm{~ms}$. Neuronal discharge rates were computed both as raw activity (epoch 2) and as relative activity defined as raw activity (epoch 2) minus background activity (epoch 1 ).

\section{Histology and reconstruction}

In the last days of recording, electrolytic microlesions $(10 \mu \mathrm{A}$ for $10 \mathrm{~s}$ ) and dye injections were made. After recording was completed, the monkey was given an overdose of sodium pentobarbital and, after respiratory block and cessation of all reflexes, transcardially perfused. Sections were made at $50-\mu \mathrm{m}$ thickness and stained alternately with cresyl violet for cytoarchitecture, and with the Gallyas method for myeloarchitecture. Electrode tracks were identified on the basis of the relative location of the penetration to the entire recorded area, the spatial relationship to other tracks and marking lesions or injections, and the depth profile during a penetration. Approximate location of each recording site on the track was determined on the basis of the distance from the above specified landmarks as well as the appearance and disappearance of gray matter. Camera lucida drawings of the relevant sections as well as two-dimensional maps of the recorded hemisphere were made as a standard procedure (Ungerleider and Desimone 1986; van Essen and Maunsell 1980).

\section{Statistics}

Differences in neuronal activity resulting from different fixation locations in the fixation paradigm or from different starting positions in the pursuit paradigm were tested for statistical significance with a distribution free analysis of variance. The effects of horizontal and vertical eye position on neuronal discharge were assumed to be independent and noninteracting. Two-dimensional linear regression analysis (least-square estimates) was applied to the mean stimulus-driven activity in the fixation paradigm and both kinds of responses, raw as well as relative activity, in the pursuit paradigm. $R^{2}$ values and the $F$ ratio were computed for validating the planar model as fit to the observed data.

\section{RESULTS}

Recordings were made from three hemispheres of three macaque monkeys. A total of 404 neurons was recorded: 354 neurons from area MST and 50 neurons from area MT. Figure 1 shows two-dimensional map reconstructions (Ungerleider and Desimone 1986; van Essen and Maunsell 1980) of the recording sites in the two male monkeys. Response characteristics as well as recording depths in individual electrode tracks had given evidence for expecting most MST recording sites of monkeys $I$ and $I I$ to be located adjacent to the ventral border of area MT and reaching throughout the fundus into the anterior bank of the STS, which was confirmed by our histological reconstructions. The vast majority of neurons in both areas responded selectively to the direction of a moving stimulus. Most area MT neurons responded preferably to moving light bars. Area MST neurons sometimes responded better to motion of random dot 

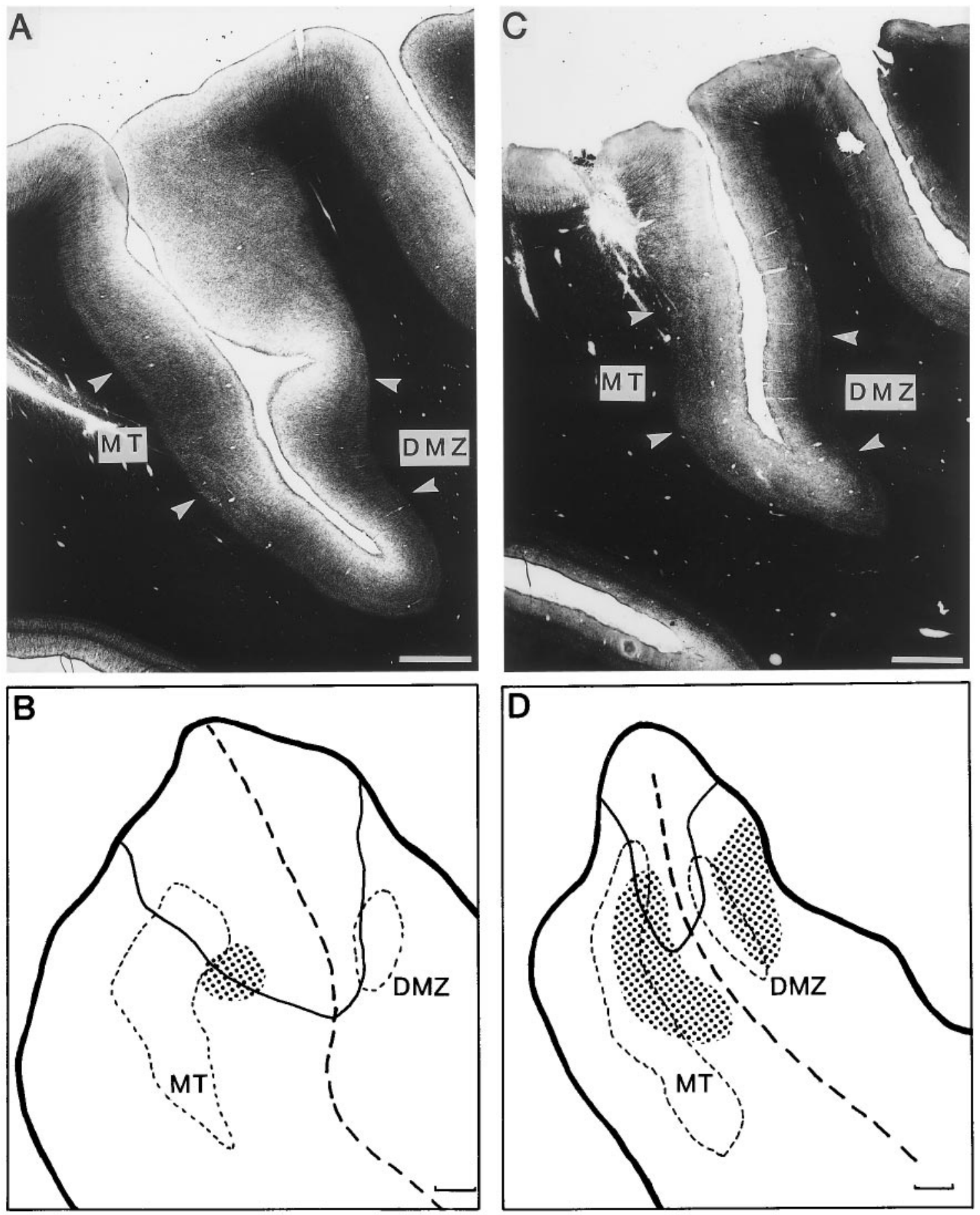

FIG. 1. Cortical regions in the male Macaca mulatta $(A$ and $B$ ) and the Macaca fascicularis $(C$ and $D)$ from which most of the data in this study were recorded. $A$ and $C$ : representative sections through the recorded area in the right superior temporal sulcus stained for myelin. Arrows: areal boarders. $B$ and $D:$ 2-dimensional maps of the right superior temporal sulcus. Thick continuous line: lip. Thick dashed line: fundus of the sulcus. Thin dashed outlines: borders of the middle temporal area (area MT) and the densely myelinated zone (DMZ) of the medial superior temporal area (area MST), respectively. Dotted areas: cortical regions where the electrophysiological recordings were performed. Thin line: layer IV line of the section shown in $A$ and $C$, respectively. Scale bar: $2 \mathrm{~mm}$.

patterns compared with light bars. On average, neurons in area MST had much larger RFs compared with area MT neurons, often extending into the ipsilateral visual hemifield. Some MST neurons proved to have RF sizes in the range of the tangent screen, i.e., from $20^{\circ}$ in the ipsilateral hemifield to $50^{\circ}$ in the contralateral hemifield or even larger (see also METHODS). Preferred stimulus speeds ranged from $5^{\circ} \%$ to $>100^{\circ} / \mathrm{s}$ for neurons from both areas.

\section{Fixation paradigm}

NEURON LEVEL. Overall, 109 neurons were tested in the fixation paradigm: 28 neurons from area MT and 81 from 


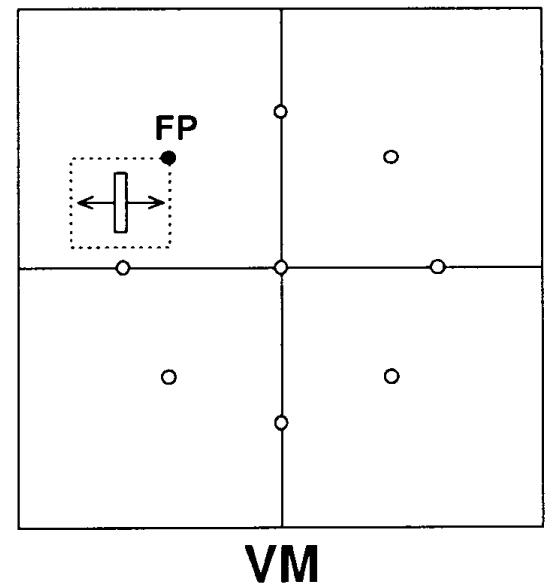

trial $n$

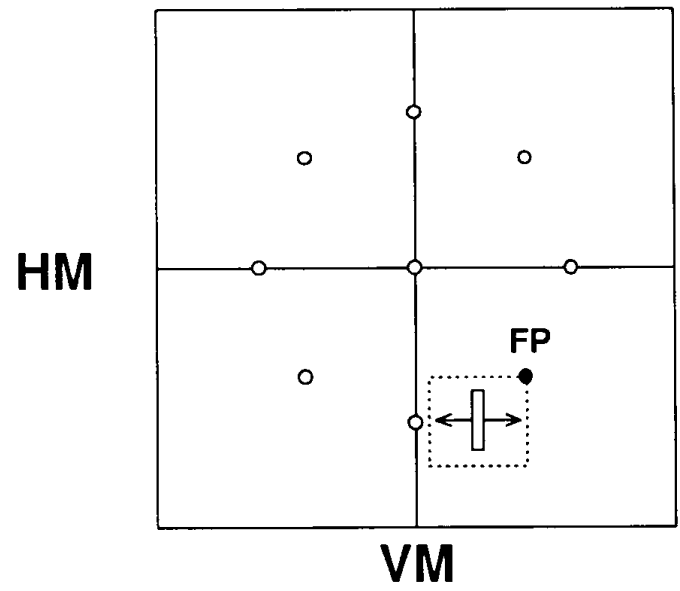

trial $n+1$
FIG. 2. Scheme of the fixation paradigm with visual stimulation. In consecutive trials $(\mathrm{n}, \mathrm{n}+1)$ the fixation point $(\mathrm{FP})$ was positioned in random order at 1 of 9 different locations on the tangent screen in front of the monkey. Clamping the stimulus to eye position allowed movement of a stimulus always across the neuron's receptive field (RF), indicated by the dotted line.
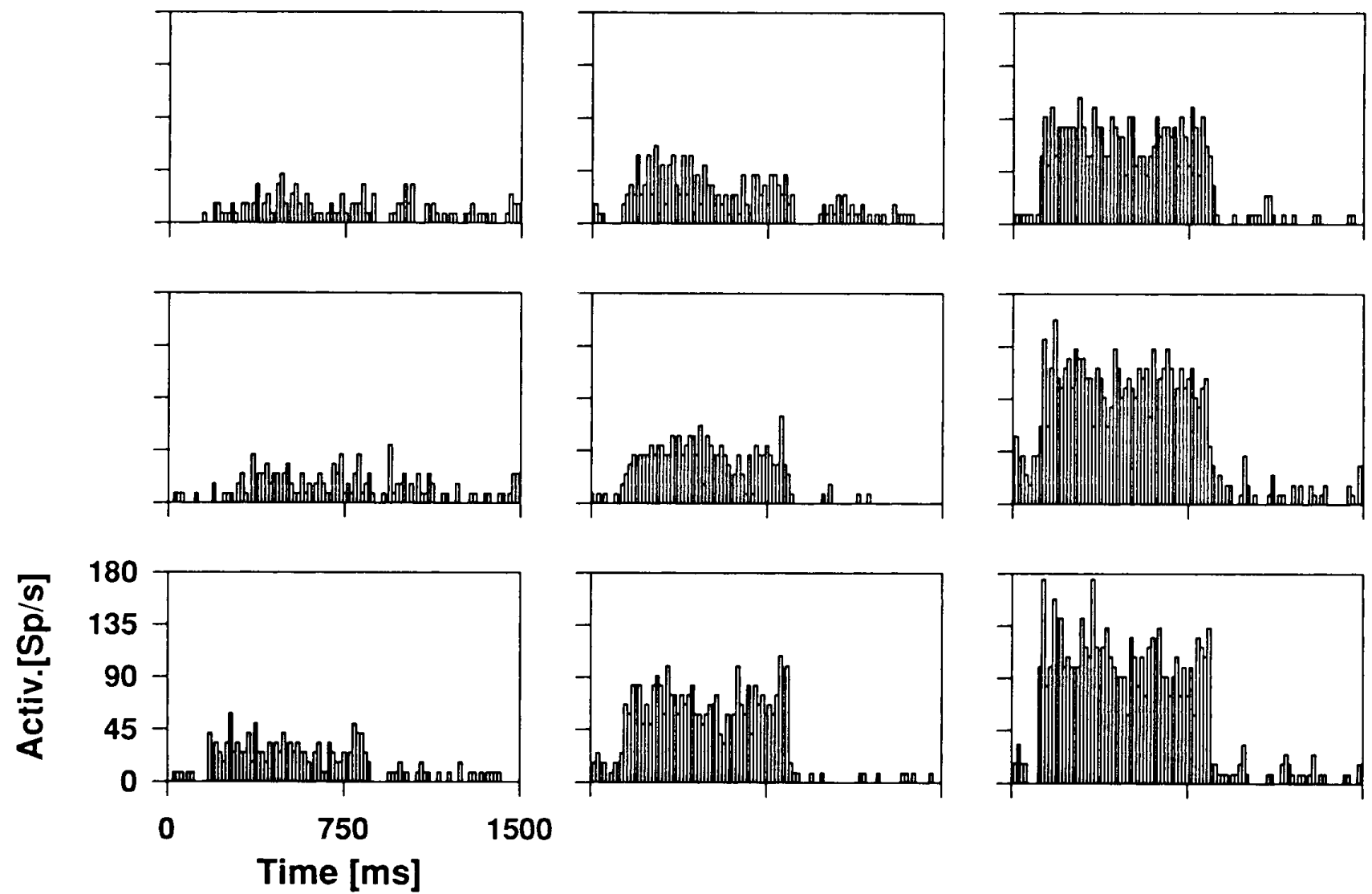

FIG. 3. Activity of an MST neuron during visual stimulation tested in the fixation paradigm. In this task the monkey had to fixate a target during visual stimulation. The RF of this neuron was extremely large $\left(100^{\circ} \times 100^{\circ}\right)$ with its geometric center near the fovea. The stimulus pattern (random dot pattern with a size of $30^{\circ} \times 30^{\circ}$ ) was moved horizontally across the central part of the RF at a velocity of $40^{\circ}$ /s with an amplitude of $\pm 15^{\circ}$. Each peristimulus time histogram (PSTH) depicts the neuronal response to movement of an optimized stimulus in the neuron's preferred direction (1st half of each PSTH) and nonpreferred direction (2nd half of each PSTH). Abscissa: stimulus duration. Ordinate: neuron's response strength. Each PSTH is located with respect to the fixation point on the screen during recording, e.g., the top left PSTH represents the stimulus response for the trials where the monkey had to fixate in the upper left, etc. Stimulus responses were best $(P<$ 0.0001 ) for fixation locations to the right of the vertical meridian. 

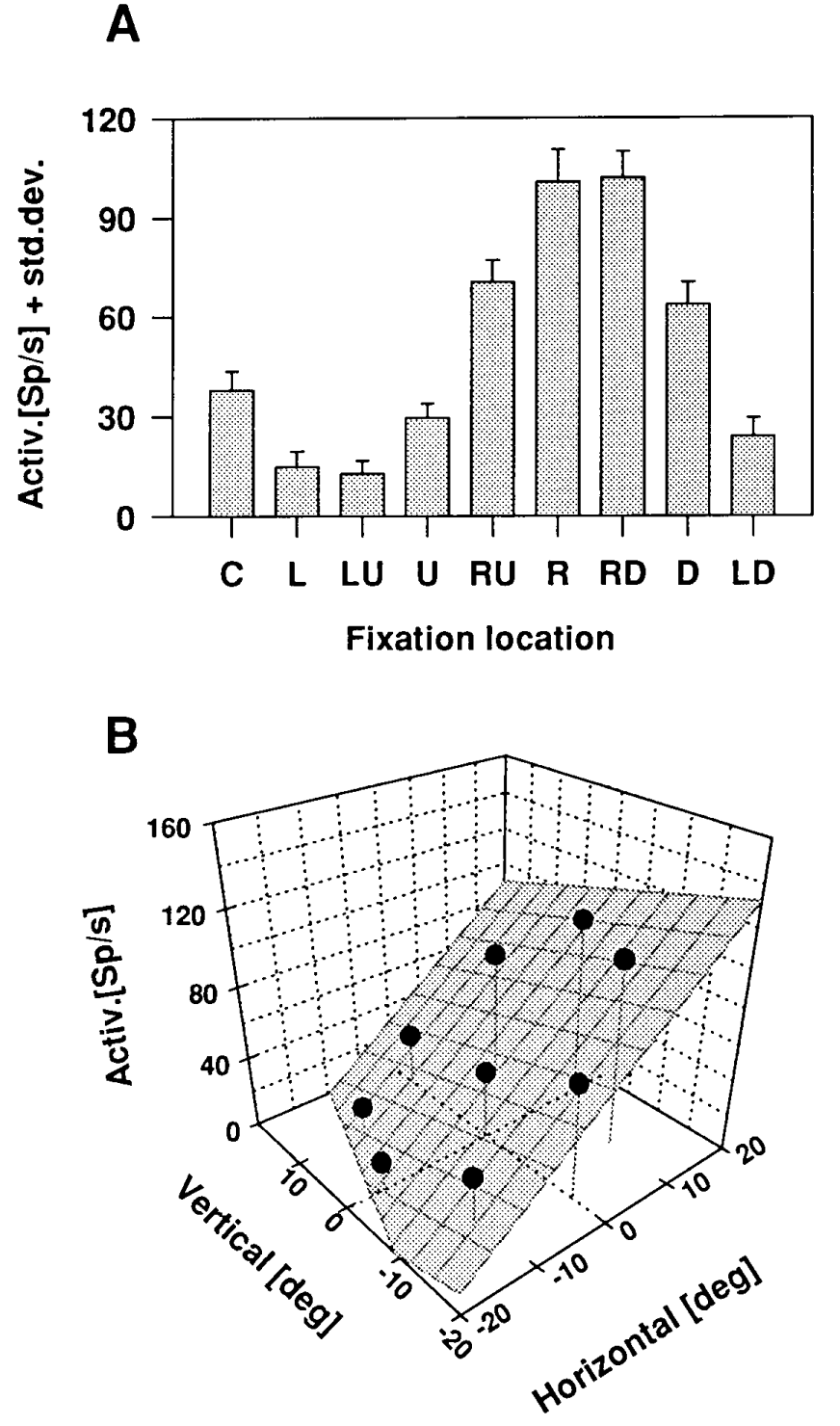

$z[$ Spikes $/ \mathrm{s}]=3.026 \mathrm{x}-1.074 \mathrm{y}+50.68$

$$
r^{2}=0.954 . p<0.0001
$$

FIG. 4. Quantification of the eye position effect on the stimulusdriven activity. A: histogram depicts mean discharges plus SDs during fixation at the 9 different locations (C, center; L, left; LU, left and up; $\mathrm{U}$, up; RU, right and up; R, right; $\mathrm{RD}$, right and down; $\mathrm{D}$, down; $\mathrm{LD}$, left and down). $B$ : 2-dimensional linear regression plane was approximated to the stimulus-induced responses. The $X-Y$ base of the cube depicts the central $\pm 20^{\circ} \times \pm 20^{\circ}$ of the screen where the monkey had to fixate. Base point of each drop line: fixation location. Height of each line: mean neuronal discharge during fixation at this location for movement of an optimized stimulus in the neuron's preferred direction. The regression plane could be fit highly significantly $(P<0.0001)$ to the observed data.

area MST. For $61 \%$ ( 17 of 28 ) of the MT neurons as well as $82 \%$ ( 66 of 81 ) of the MST neurons, the stimulus-induced activity was modulated significantly $(P<0.05)$ by the position of the eyes in the orbit.

A graphic illustration of the fixation paradigm is shown in Fig. 2. In consecutive trials, the fixation point (FP) was located in random order at one of nine different locations on the screen. During each trial, an optimized visual stimulus was moved across the neuron's RF (indicated by the dotted line) in its preferred and nonpreferred direction. The monitored eye position signal was superimposed onto the stimulus driving signal, which allowed stimulation of always the same retinal location. An example for the influence of the position of the eyes in the orbit on the stimulus-driven response of an MST neuron is shown in Fig. 3. PSTHs shown are arranged according to the fixation location on the screen. Thus in each PSTH the neuron's response to stimulus movement of an optimized stimulus in the preferred direction (1st half) and

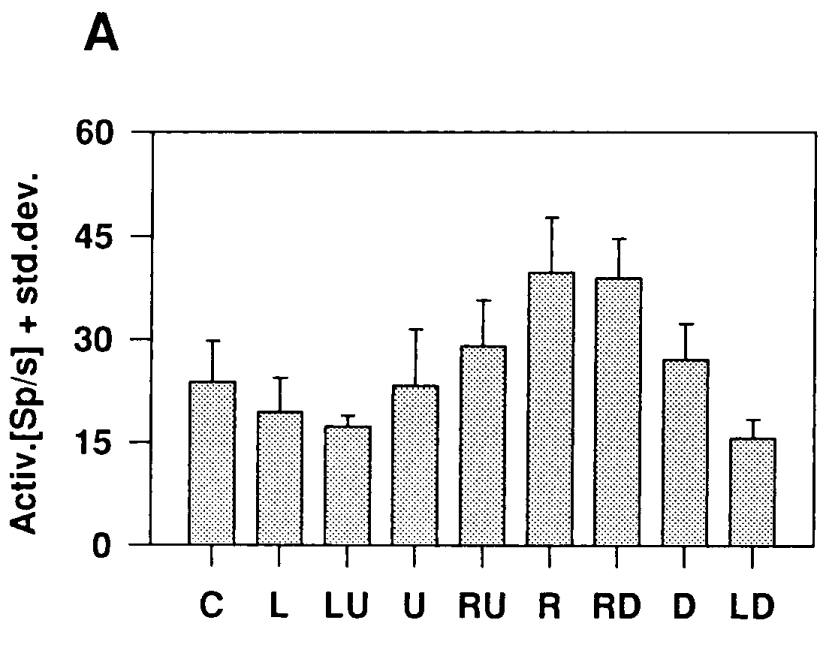

Fixation location

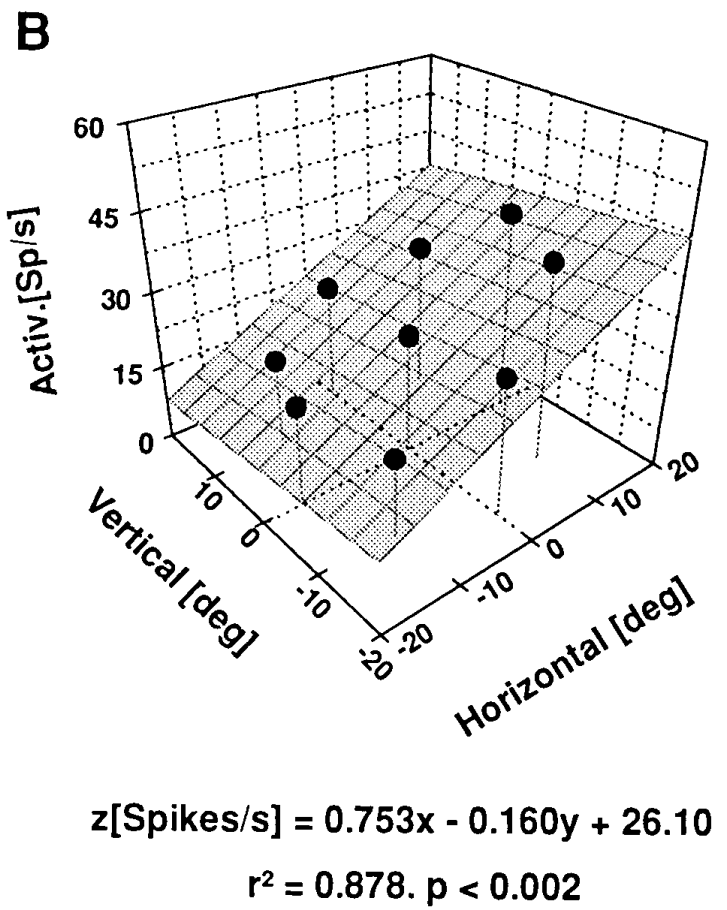

FIG. 5. Activity of an MST neuron in the fixation paradigm without visual stimulation. In this task the monkey had to fixate a spot of light in an otherwise dark room without additional visual stimulation. Even in darkness, ongoing neuronal discharges were modulated by eye position: activity was highest for fixation locations to the right of the vertical meridian. $A$ : data depicted as in Fig. 4. B: 2-dimensional regression plane provided a significant $(P<0.002)$ approximation to the neuronal discharges. 

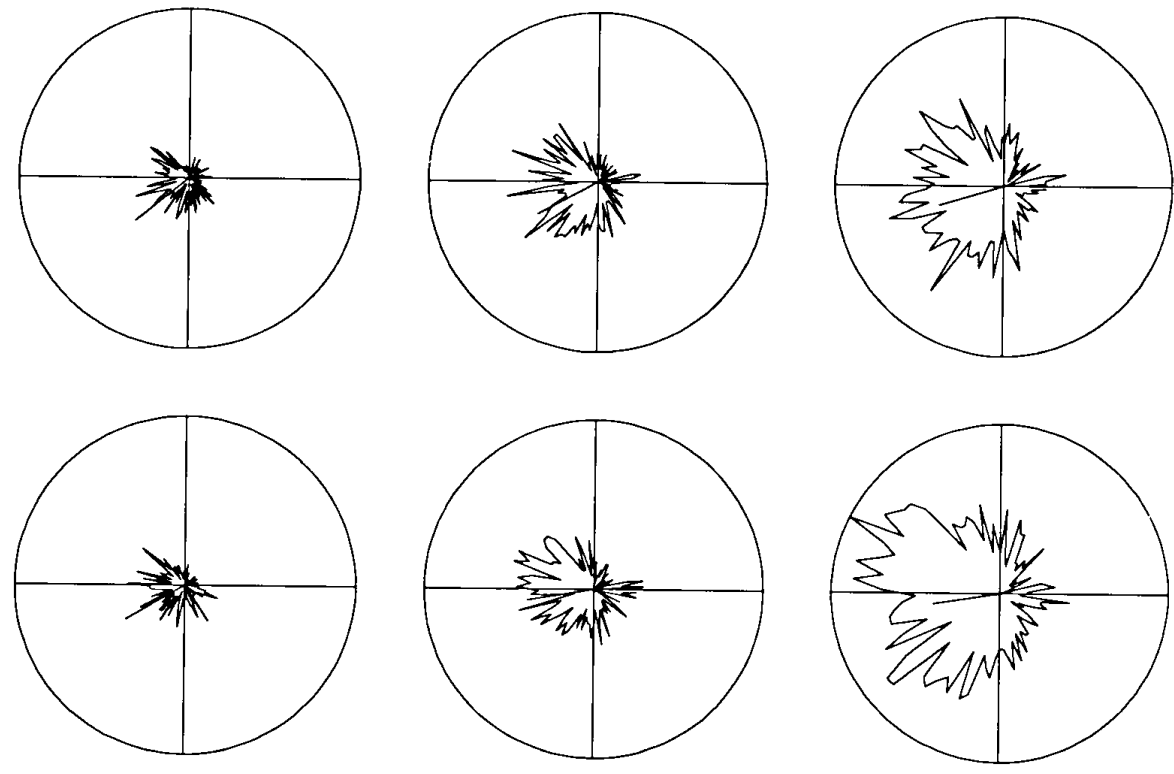

FIG. 6. Continuous mapping of directional selectivity in the fixation paradigm. During fixation at different locations, the monitored eye position signal was superimposed onto the stimulus signal. A random dot pattern $\left(30^{\circ} \times 30^{\circ}\right)$ was moved in a 2 dimensional plane along a circular pathway in each trial. Responses are plotted in the stimulus plane. The origin location of each polar plot represents the fixation location on the screen. Although response strength is varied by eye position (strongest responses for fixation on the right), preferred direction (represented by a solid line pointing away from the center of each polar plot)
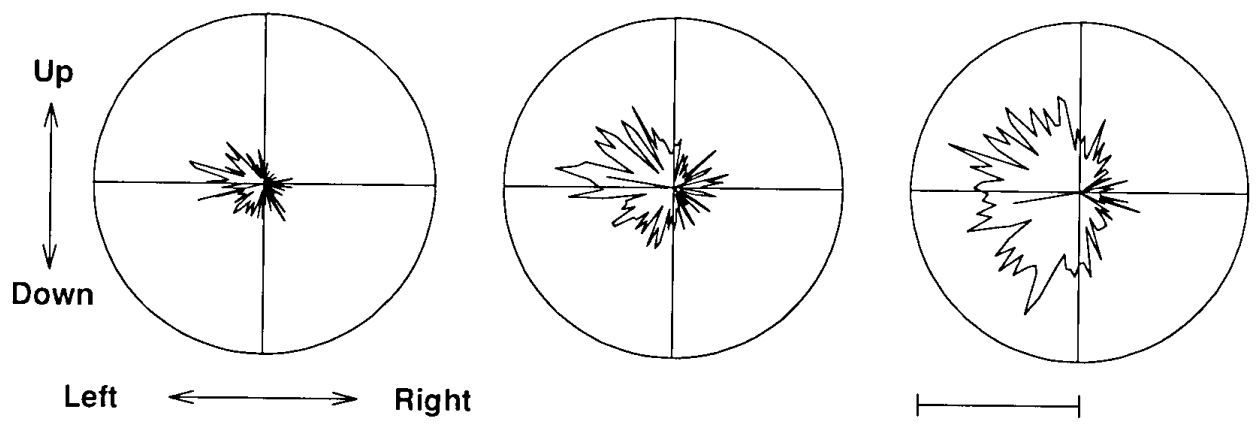
keeps nearly constant, pointing to the left during fixation at all different locations. In each polar plot the radius of the outer circle corresponds to a cell discharge of 200 spikes/s.

Left

Right

200 Spikes/s

nonpreferred direction (2nd half) during fixation at that particular location is shown, e.g., the PSTH at top left represents the stimulus responses for trials where the monkey had to fixate in the upper left. For this individual MST neuron, responses were best when the animal fixated locations right of the vertical meridian $(P<0.0001$; rightmost PSTH column). Variation of vertical eye position led to an increase in activity for downward-directed fixations. If there was any influence of small changes in stimulus contrast across the

TABLE 1. Preferred stimulus directions at different eye positions

\begin{tabular}{lcc}
\hline \hline Eye Position & PD, deg & F, deg \\
\hline 0,0 (Center) & 189.3 & - \\
15,0 & 188.5 & 0.8 \\
$-15,0$ & 210.0 & -20.7 \\
0,15 & 207.8 & -18.5 \\
$0,-15$ & 171.1 & 18.2 \\
$10,6,10.6$ & 196.5 & -7.2 \\
$-10.6,10.6$ & 218.7 & -29.4 \\
$-10.6,-10.6$ & 194.3 & -5.0 \\
$10.6,-10.6$ & 189.7 & -0.4 \\
\hline
\end{tabular}

First column assigns the 9 different eye positions. Second column: values for the computed preferred direction for each eye position. Third column: angular difference between the preferred stimulus directions during fixation at the center and each eccentric eye position. PD, preferred direction. F, angular difference. screen, one would expect the strongest response for all neurons to be in the center. However, we observed the best response for 64 of the $66(97 \%)$ of eye-position-sensitive neurons at eccentric fixation locations, i.e., only 2 neurons (3\%) revealed a response maximum during central fixation. This value is clearly below chance level (11\%) and therefore rules out any eventually occurring variations in stimulus contrast as origin of the changes in neuronal responsiveness.

To quantify the modulatory effect of eye position on the stimulus-induced response, we employed a two-dimensional linear regression analysis. The result of this computation is shown in Fig. 4. The histogram (Fig. 4A) depicts mean responses plus SDs for stimulus movement into the preferred direction during fixation at different locations. The $X-Y$ base of the cube (Fig. 4B) represents the central $\pm 20^{\circ} \times \pm 20^{\circ}$ of the tangent screen where the monkey had to fixate. The base point of each drop line assigns the fixation location on the screen. The height of each drop line depicts the mean preferred-directionstimulus-driven activity during fixation at this location. Mean responses were strongest for fixations to the right and smallest for fixations to the left of the vertical meridian. A two-dimensional linear regression function, represented by the shaded plane, could be fit significantly $(P<0.0001)$ to the stimulus-induced responses.

The neuron was further tested during fixation without presenting any other visual stimulus, i.e., the screen the animal 


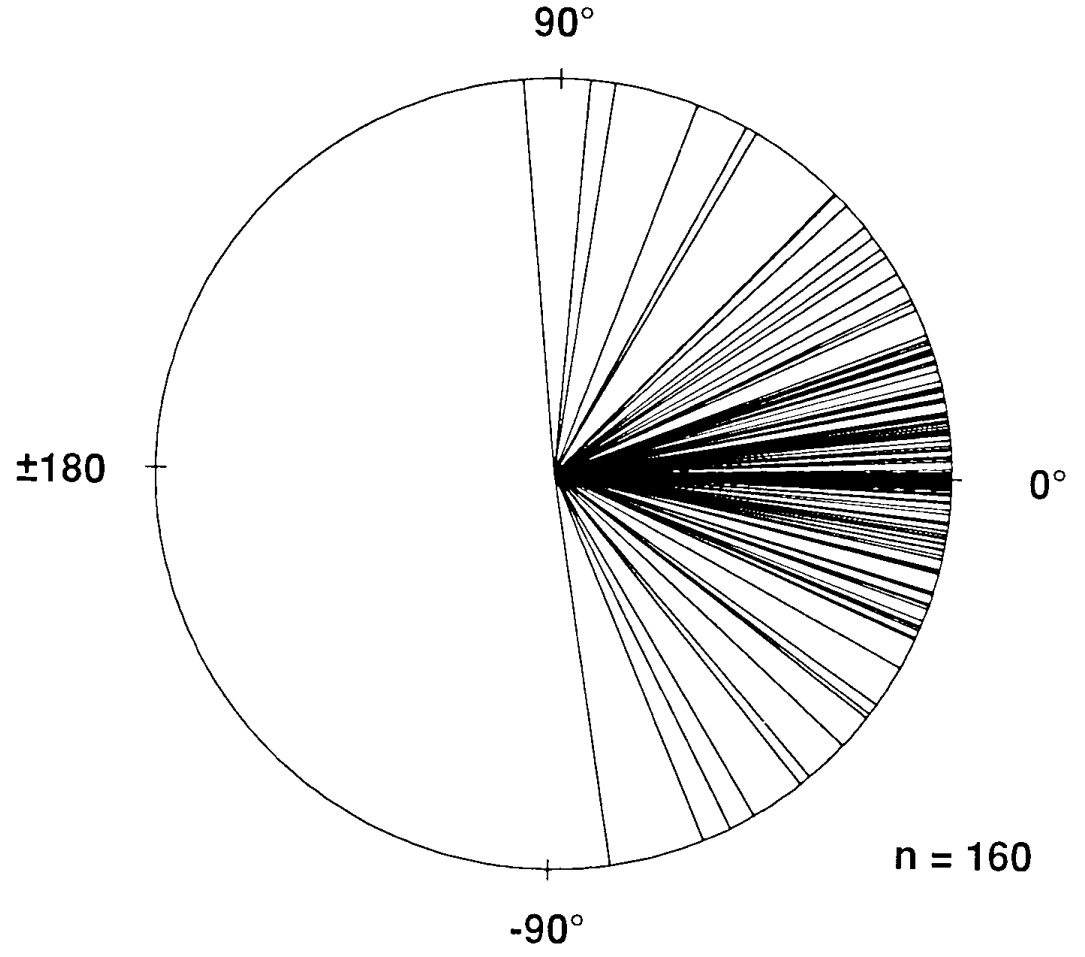

FIG. 7. Constancy of directional selectivity tested for area MST. Each line depicts the difference between the preferred stimulus direction during fixation straight ahead and during eccentric fixation. For details of this computation see RESULTS. Cardinal directional selectivity proved to be unaffected by the variation of gaze direction.
Area MT

A
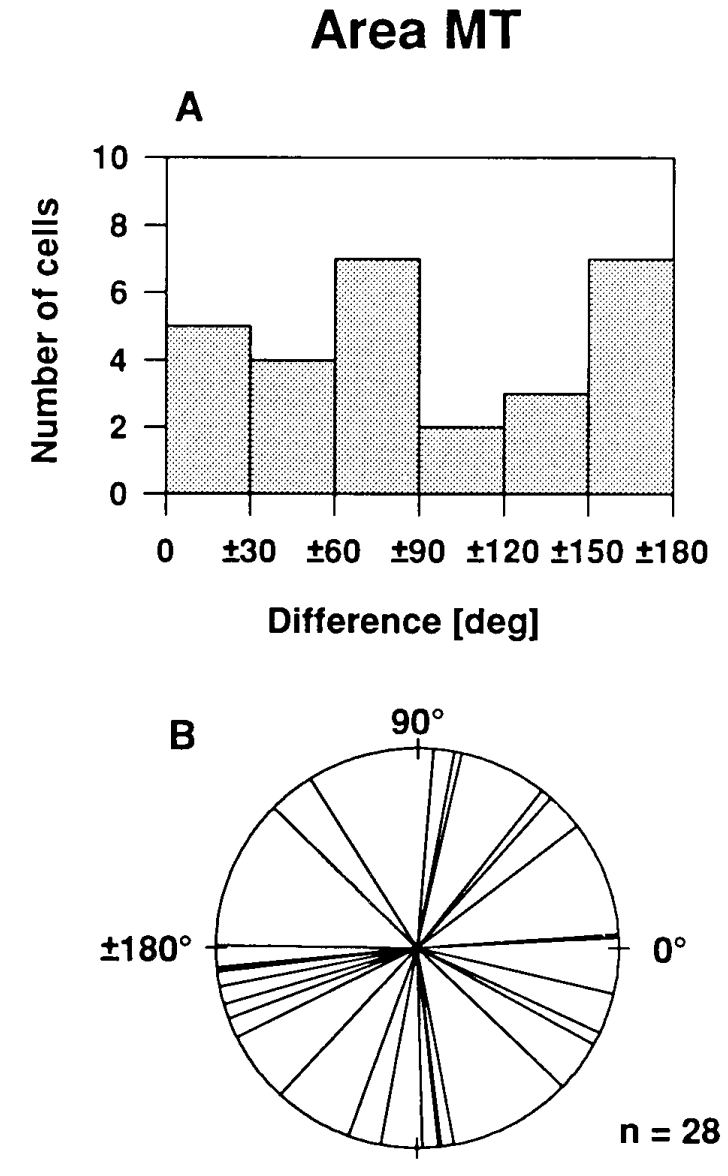

$-90^{\circ}$

FIG. 8. Difference between preferred stimulus direction and the direction of the gradient of the linear regression plane. For details of computation see text. Angular differences are represented in histograms $(A$ and $C)$ and as circular scatter plots $(B$ and $D)$. Statistical analysis did not reveal any significant correlation between both directions ( $\chi^{2}$ test), either for area MT or area MST. 
looked at was totally dark except for the fixation target (luminance $<0.0001 \mathrm{~cd} / \mathrm{m}^{2}$ ). As can be seen in Fig. 5, the activity of this individual neuron was influenced even by the position of the eyes in the orbit per se. Activity was highest $(P<$ 0.0001 ) for fixation locations right of the vertical meridian, which corresponds to the result observed for visual stimulation during fixation. A regression plane provided a significant approximation $(P<0.001)$ to the neuronal discharges.

In a final step, this neuron was tested for a variation of its preferred stimulus direction with varying eye position. During each trial, the stimulus pattern was moved along a circular path across the RF. The result of this directional tuning test is shown in Fig. 6. Each polar plot represents the neuronal response for stimulation during fixation at a specific location. The polar plot at bottom right thus depicts the stimulus response for moving the pattern along the circular pathway during fixation in the lower right $([X, Y]=$ $\left.\left[10.6^{\circ},-10.6^{\circ}\right]\right)$. The overall response strength varied with the fixation location. However, the response peak remained invariable, pointing approximately to the left within each individual response plot, i.e., the directional selectivity of the neuron was unaffected by varying eye position. The neuron's preferred direction (computed by means of the SDO analysis) for each eye position is depicted graphically by a single solid line in each polar plot.

SYSTEM LEVEL. Overall, 20 MST neurons from the second monkey were tested systematically for an influence of the position of the eyes in the orbit on their preferred stimulus direction. As in the example for the single neuron shown before, for each individual neuron the preferred directions for stimulation during fixation at different locations were computed. We defined the preferred stimulus direction for fixation at the screen center $\left([X, Y]=\left[0^{\circ}, 0^{\circ}\right]\right)$ as $P D_{\text {Center }}$ and the eight preferred directions obtained during eccentric fixation $\left([X, Y]=\left[ \pm 10.6^{\circ}, \pm 10.6^{\circ}\right],\left[ \pm 15.0^{\circ}, 0.0^{\circ}\right]\right.$, $\left.\left[0.0^{\circ}, \pm 15.0^{\circ}\right]\right)$ as $P D_{\mathrm{Ecc}}(i) . P D_{\mathrm{Center}}$ was defined as the reference value. For each individual neuron we then computed the eight angular differences between $P D_{\mathrm{Center}}$ and $P D_{\mathrm{Ecc}}(i)$ as

$$
F(i)=P D_{\text {Center }}-P D_{\mathrm{Ecc}}(i)
$$

The numerical values of angular differences for the example shown before (Fig. 6) are given in Table 1. A graphic representation of the outcome of this computation for the ensemble of neurons $(n=20)$ tested is shown in Fig. 7. The angle of a single line represents one angular difference $F(i)$ of a single neuron. The mean difference for all neurons tested was $-2.7 \pm 24.9^{\circ}$ (mean $\left.\pm \mathrm{SD}\right)$. This result indicates that for the population of neurons the directional tuning is not influenced by eye position.

In the above example the neuron's preferred stimulus direction was opposite to the direction of the gradient of the approximated response plane, i.e., the direction of the steepest slope of the plane. Stimulus response was best for more rightward fixations, whereas the preferred stimulus direction was to the left. We wanted to test whether there exists a general relationship between these two response parameters.

Preferred stimulus directions $D_{\text {StimPD }}$ were uniformly distributed in both area MT ( $\chi^{2}$ test: $\left.P>0.5\right)$ and area MST $\left(\chi^{2}\right.$ test: $\left.P>0.8\right)$, respectively. The direction of the gradient $D_{\text {GradFix }}$ of an individual regression plane was computed as

$$
D_{\text {GradFix }}=\arctan (b / a)
$$

where $a$ and $b$ represent the horizontal and vertical slopes of the plane, respectively. We computed for each individual
Area MT

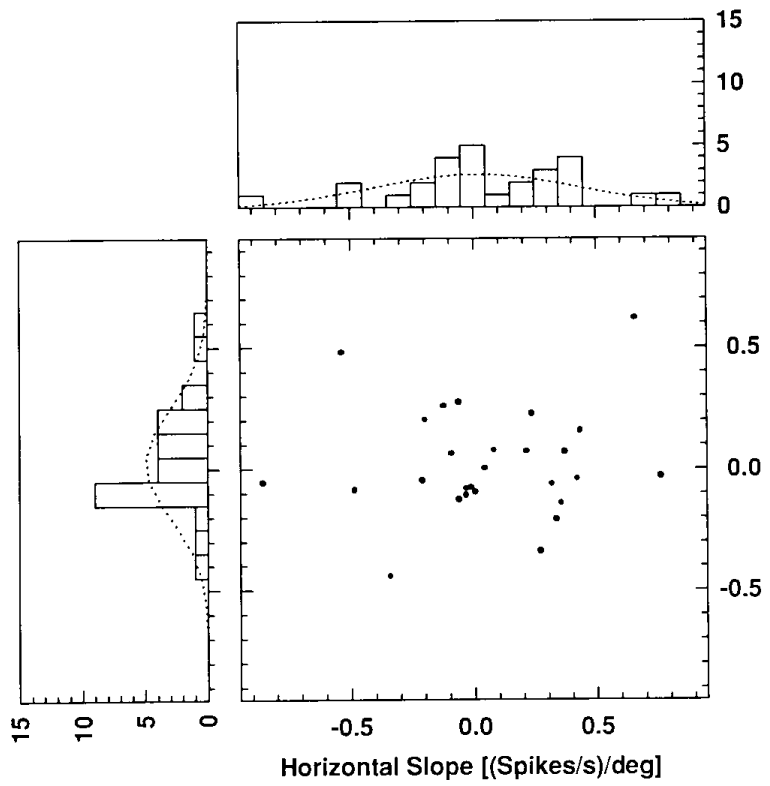

Area MST
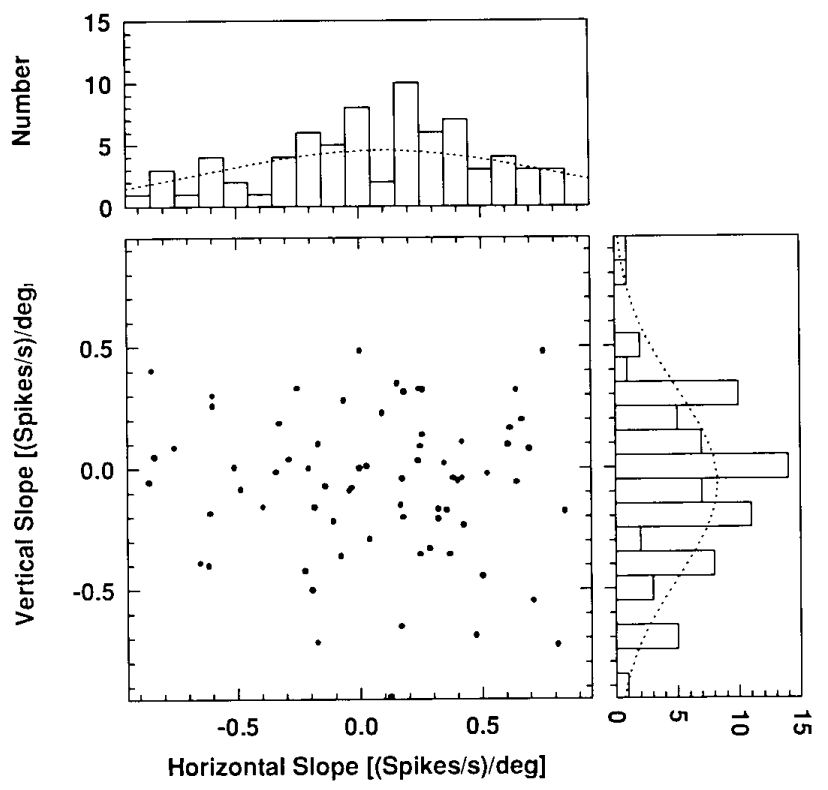

FIG. 9. Distribution of the gradients of the regression planes for areas MT and MST. Middle: gradient of an individual linear regression plane, treated as a 2-dimensional vector, is represented by a single data point. Statistical analysis proved the directions of the gradients for both areas to be uniformly distributed. A normal distribution (represented by a dotted line within each histogram plot) turned out to be the best fit to the values of the horizontal and vertical slopes for both area MT and MST, respectively. 
Area MT

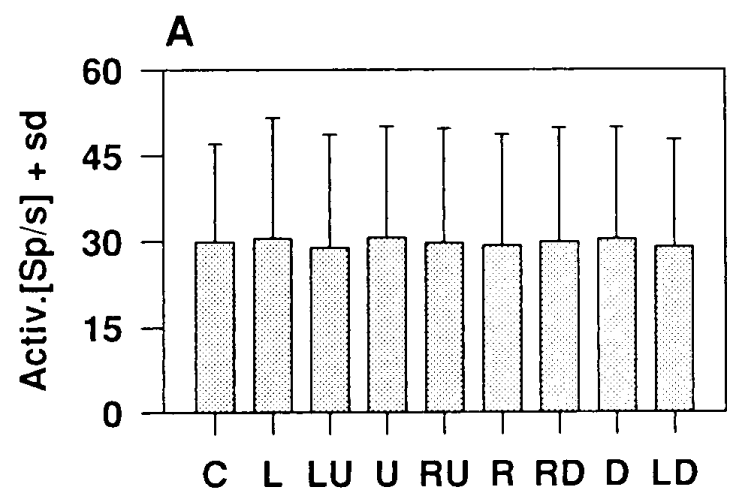

Fixation location

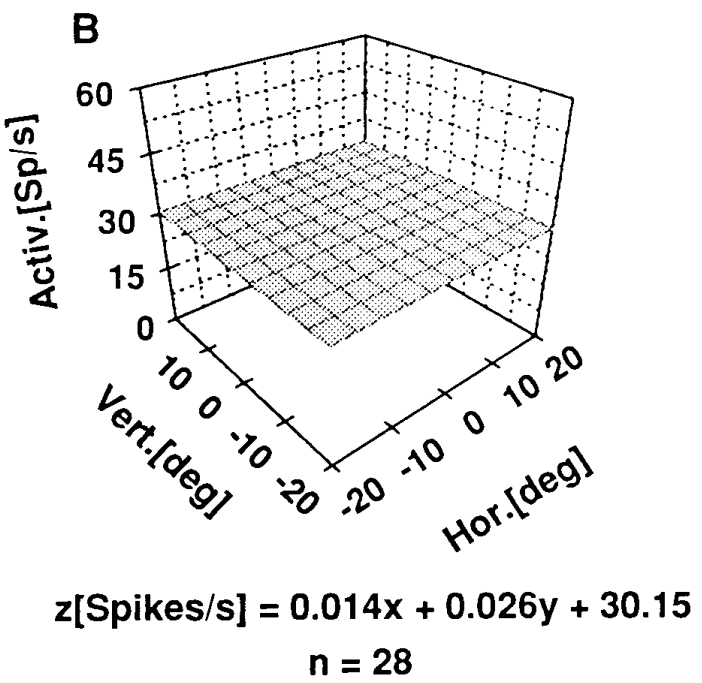

Area MST

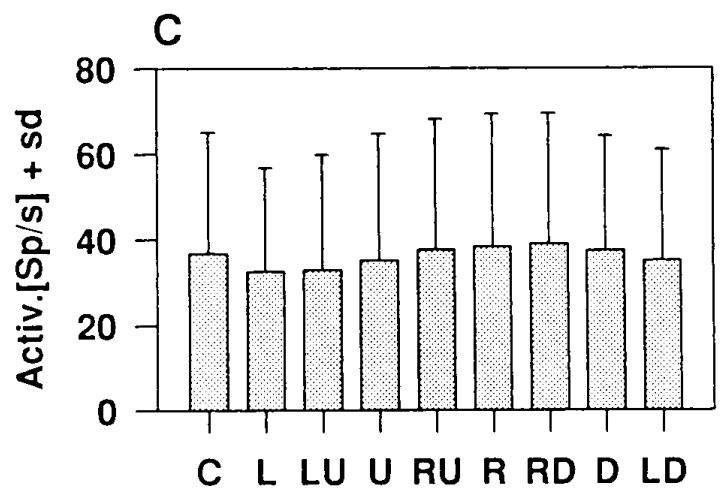

Fixation location

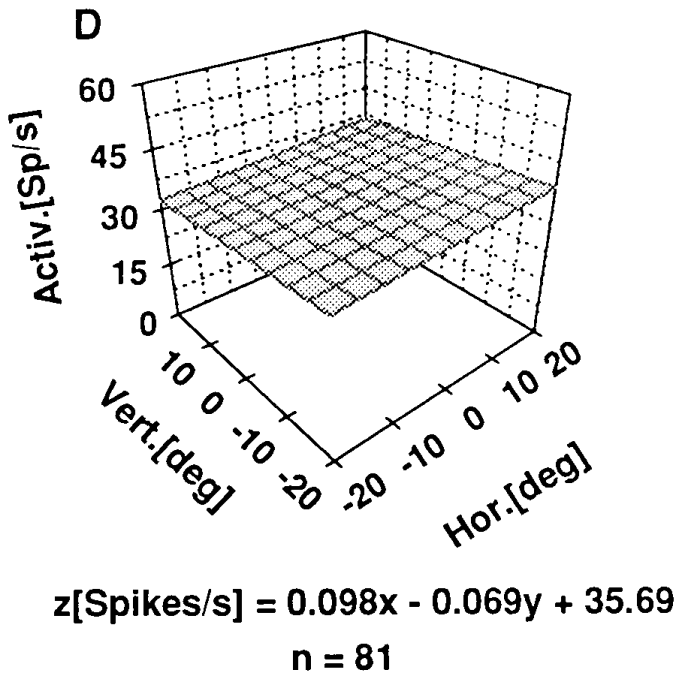

FIG. 10. Population response for neurons from areas MT and MST tested in the fixation paradigm. Histograms: mean stimulus-driven responses plus SDs during fixation at different locations for areas MT $(A)$ and MST $(C)$. Discharges during fixation at different locations were not significantly different, implying the modulatory effect of eye position to be evenly distributed at the population level. The population response planes ( $B$ and $D$ ) were obtained by averaging all linear regression planes.

neuron $(i)$ the angular difference $Y(i)$ between its preferred stimulus direction $D_{\mathrm{StimPD}}(i)$ and the direction of the regression plane gradient $D_{\text {GradFix }}(i)$ as

$$
Y(i)=D_{\mathrm{StimPD}}(i)-D_{\text {GradFix }}(i)
$$

As can be seen in Fig. 8 for areas MT and MST, both directions did not tend to correlate. In Fig. 8, $B$ (area MT) and $D$ (area MST), the angle of each line represents the angular difference $Y(i)$ for an individual neuron $(i)$. Frequency histograms of the distribution of the absolute values of the angular differences are shown in Fig. 8, A (area MT) and $C$ (area MST). The qualitative estimation that both directions were not correlated with each other could be proved to be statistically significant $\left(\chi^{2}\right.$ test: $P>0.3$ for area MT and $P>0.4$ for area MST).

Two-dimensional regression planes were approximated to the discharges of the neurons for the nine eye positions. For $53.5 \%$ (15 of 28) of the MT neurons and $60.5 \%$ (49 of 81 ) of the MST neurons the correlation coefficient $R$ of the respective regression plane was $>0.795$, therefore validating the planar model at a value of $P<0.05$. The distributions of the gradients of the approximated regression planes for both area MT and MST, respectively, are shown in Fig. 9. In the two-dimensional illustrations in the middle, each data point represents the gradient of one individual regression plane. Statistical analysis for both populations did reveal that the distribution of the directions of the gradients did not deviate significantly from a uniform distribution $(P>0.15$ for area MT and $P>0.2$ for area MST). The histograms above and to the right of the illustrations in the middle represent the distribution of the one-dimensional horizontal (top) and vertical (right) slopes of the regression planes. A normal distribution, which is represented by a dotted curve within each histogram plot, appeared to be the best approximation for these distributions.

To test for a resulting net effect of the position of the eyes in the orbit on the activity of a whole ensemble of neurons within both areas, we computed the mean stimulusinduced response for the population of all neurons tested. This response was obtained by computing the mean of all linear regression planes fit to the single-cell data in area MT and area MST. The result is shown in Fig. 10. For both areas 

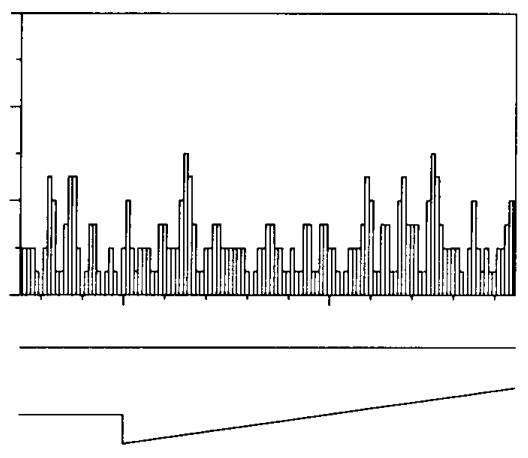
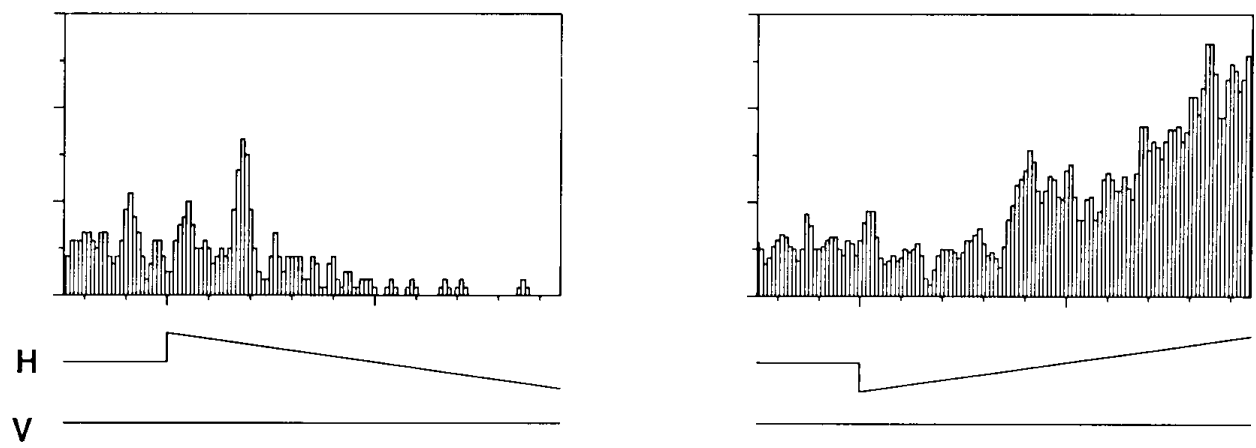

FIG. 11. Pursuit-related responses of an MST neuron tested in the step ramp paradigm. In each PSTH neuronal discharge is shown from $500 \mathrm{~ms}$ after the beginning of each trial until the end of the smooth pursuit phase. Each PSTH is located with respect to the movement direction of the target. This individual neuron revealed a continuously increasing activity for pursuit to the right as well as a continuous decrease for pursuit to the left.

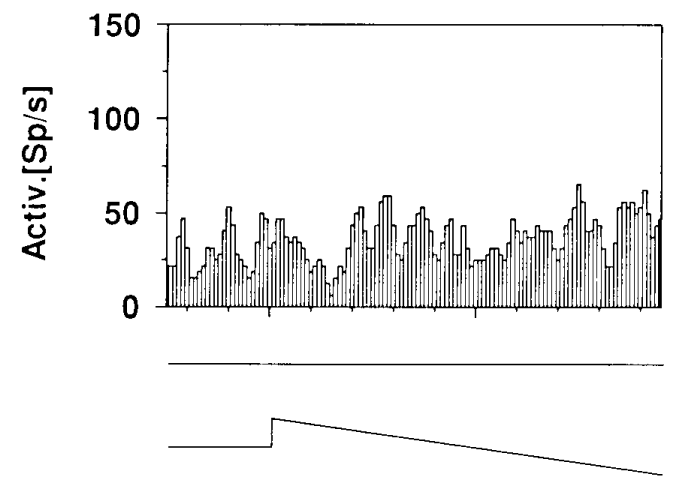

$200 \mathrm{~ms} / \mathrm{div}$

the mean response plane turned out to be essentially flat, indicating that the modulatory effect of eye position that could be observed at the single-cell level was balanced out at the population level. Statistical analysis revealed that the mean discharge values at the different fixation locations did not differ significantly from each other (analysis of variance: $P>0.999$ for area MT and $P>0.7$ for area MST).

\section{Pursuit paradigm}

NEURON LEVEL. A total of 354 neurons was tested for an increased discharge during smooth tracking eye movements: 40 neurons from area MT and 314 from area MST. Fourteen (35\%) of the MT neurons as well as $154(48 \%)$ of the MST neurons exhibited a direction-specific response when studied in the step ramp paradigm. For 78\% (11 of 14) of the MT neurons as well as $80 \%$ (124 of 154) of the MST neurons, an influence of eye position on the activity during pursuit could be observed.

Figure 11 shows the activity of an MST neuron when tested in the step ramp paradigm. The PSTHs are arranged with respect to the movement direction of the target: the bottom PSTH represents the neuronal activity for the trials where the target stepped upward and then moved downward, etc. Examination of the neuronal responses revealed that this individual neuron preferred pursuit movement to the right.

The result of testing this neuron in the pursuit paradigm, where the target always moved to the right starting from different locations, is shown in Fig. 12. Each PSTH is located with respect to the starting location of pursuit. In each PSTH neuronal responses are aligned to the onset of the saccade, which precedes the smooth pursuit phase. For this neuron, pursuit responses were best for starting locations to the right of the vertical meridian and weakest for starting locations to the left of the vertical meridian $(P<0.0001)$. Variation of vertical eye position had no significant effect on the neuronal activity. Two-dimensional linear regression analysis again was used to quantify the observed effect. The result of this computation is shown in Fig. 13. The histogram (Fig. 13A) depicts the mean discharges plus SDs for pursuit starting 

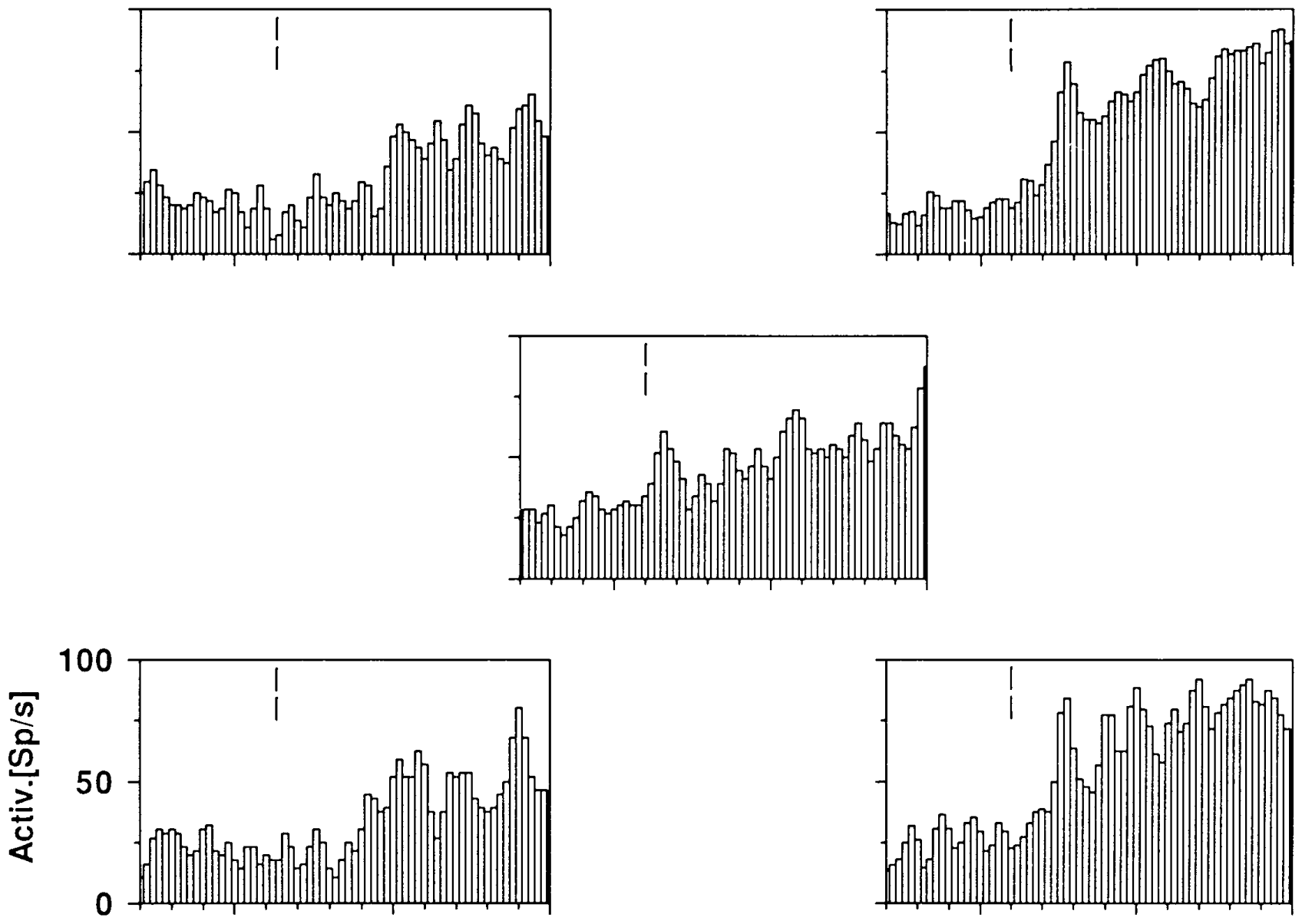

\section{$100 \mathrm{~ms} / \mathrm{div}$}

FIG. 12. Neuronal discharges during the pursuit paradigm. The target started in random order from 1 of 5 different locations and then always moved to the right. Neuronal discharges are aligned to the onset of the catch-up saccade, which precedes each smooth pursuit. Saccade onset is given by the vertical dashed line within each PSTH. For this individual neuron pursuit-related responses were best for starting locations to the right of the vertical meridian (rightmost PSTHs).

from the different locations. The $X-Y$ base of the cube (Fig. $13 B$ ) depicts the central $\pm 20^{\circ} \times \pm 20^{\circ}$ of the screen where the monkey had to pursue the target. The base point of each drop line represents the starting point of pursuit and the height of each line indicates the mean neuronal activity (raw activity) for pursuit starting from this location. A two-dimensional linear regression plane could be approximated significantly $(P<0.005)$ to the data.

SYSTEM LEVEL. In the example given above, the neuron's preferred pursuit direction $D_{\text {PurspD }}$ did correlate with the direction of the gradient $D_{\text {GradPurs }}$ of the regression plane approximated to the data obtained in the pursuit paradigm. To test whether this finding holds true as a general response characteristic for area MT and MST neurons, we computed the distribution of the difference of both directions.

Preferred pursuit directions $D_{\text {PursPD }}$ were uniformly distributed in both area MT $\left(\chi^{2}\right.$ test: $\left.P>0.4\right)$ and area MST $\left(\chi^{2}\right.$ test: $P>0.7)$. The direction of the gradient $D_{\text {GradPurs }}$ of a regression plane was computed as

$$
D_{\text {GradPurs }}=\arctan (b / a)
$$

with $a$ and $b$ the horizontal and vertical slopes of the plane, respectively. For each individual neuron $(i)$ the angular difference $D(i)$ between the preferred pursuit direction $D_{\text {PursD }}(i)$ and the direction of the approximated regression plane $D_{\text {GradPurs }}(i)$ was computed as

$$
D(i)=D_{\mathrm{PursPD}}(i)-D_{\text {GradPurs }}(i)
$$

In the circular scatter plot of Fig. 14A, each single line represents the angular difference $D(i)$ for an individual MST neuron. The very same data were classified in a histogram of absolute values of angular differences (Fig. 14B). Quantitative analysis for both areas (MT and MST) did not reveal any correlation between both directions ( $\chi^{2}$ test: $P>0.8$ for areas MT and MST).

Overall, 86 neurons were tested in both the pursuit and the fixation paradigm, respectively: 8 neurons from area MT and 78 neurons from area MST. To quantify any possible correlation between the modulatory effects acting on the neurons in both paradigms, we compared the directions of the response plane gradients observed in the fixation para$\operatorname{digm}\left(D_{\text {GradFix }}\right)$ and the pursuit paradigm $\left(D_{\text {GradPurs }}\right)$. Both directions tended to be correlated significantly for area MST $\left(\chi^{2}\right.$ test: $\left.P<0.05\right)$. For an individual neuron $(i)$ the angular 

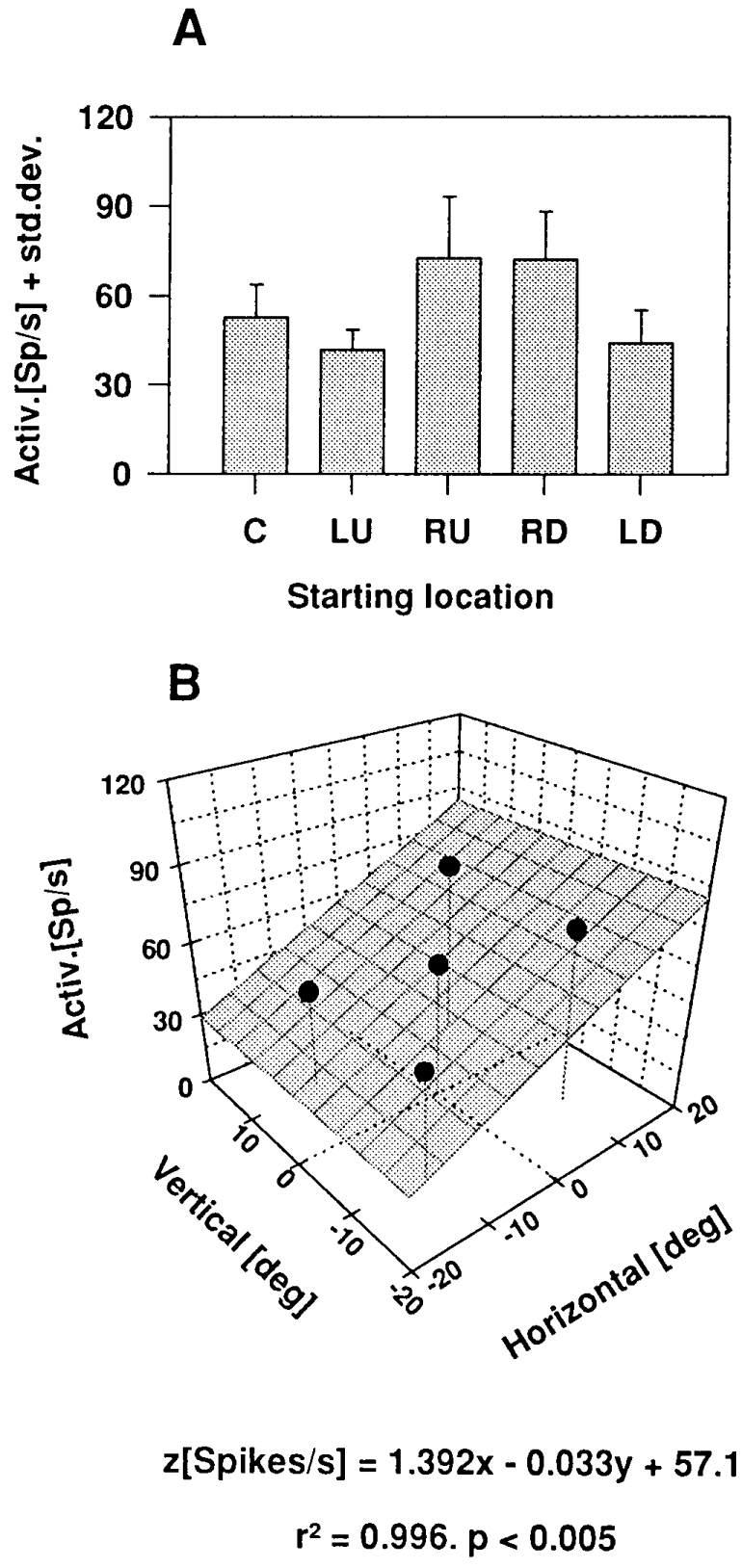

FIG. 13. Quantification of the eye position effect. $A$ : histogram depicts mean discharges plus SDs for the different starting locations of the pursuit target. $B$ : approximation of a 2-dimensional linear regression plane to the pursuit-related responses. The $X-Y$ base represents the central $\pm 20^{\circ} \times \pm 20^{\circ}$ of the screen. Base point of each drop line: starting location of pursuit. Height of each line: mean raw activity as defined in METHODS. A linear regression plane could be fit significantly $(P<0.005)$.

difference $\tilde{o}(i)$ between $D_{\text {GradPurs }}(i)$ and $D_{\text {GradFix }}(i)$ was computed as

$$
\tilde{o}(i)=D_{\text {GradPurs }}(i)-D_{\text {GradFix }}(i)
$$

For $37 \%$ (3 of 8 ) of the MT neurons as well as 33\% (26 of 78 ) of the MST neurons the angular difference was less than $\pm 30^{\circ}$. Furthermore, for $75 \%$ of the MT neurons ( 6 of 8 ) and $70 \%$ (55 of 78) of the MST neurons both gradient directions pointed at least into the same hemifield. Figure 15 shows a circular scatter plot (Fig. 15A) of the angular differences $\tilde{\sigma}(i)$ as well as a histogram of this distribution (Fig. 15B) for area MST.
We also tested for an eventually existing relationship between RF characteristics and the influence of eye position. Therefore we computed for both RF size and RF eccentricity, respectively, a correlation with the slope of the eye position regression planes. The slope $S(i)$ of an individual regression plane was computed as

$$
S(i)=\operatorname{sqrt}\left(a^{2}+b^{2}\right)
$$

with $a$ and $b$ the slopes in horizontal and vertical direction, respectively. If a neuron was tested in both paradigms, the regression plane with the steeper slope was chosen for computation. As Fig. 16 shows, the slope of the regression planes was clearly not correlated either with RF size or with RF eccentricity, either in area MT or in area MST.

We tested also for a correlation between the direction of the gradient of the regression plane $D_{\text {Grad }}$ (either $D_{\text {GradFix }}$ or $D_{\text {GradPurs }}$, as defined above) and the direction of the RF eccentricity $D_{\text {RF-Ecc }}$. For an individual neuron $(i)$ the latter was defined as the direction of the line between the fixation point $\left([X, Y]=\left[0^{\circ}, 0^{\circ}\right]\right)$ and the geometric center of the RF $([X, Y]=[X(i), Y(i)])$

$$
D_{\mathrm{RF}-\mathrm{Ecc}}=\arctan [Y(i) / X(i)]
$$

All MT and MST neurons were recorded from right hemi-

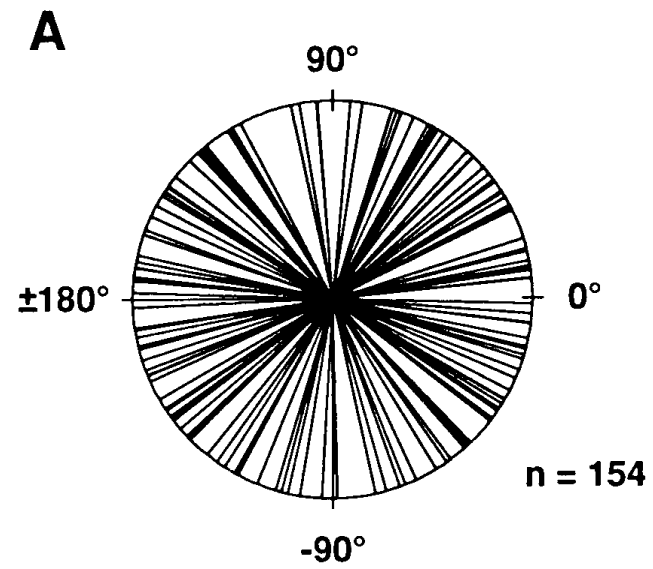

B

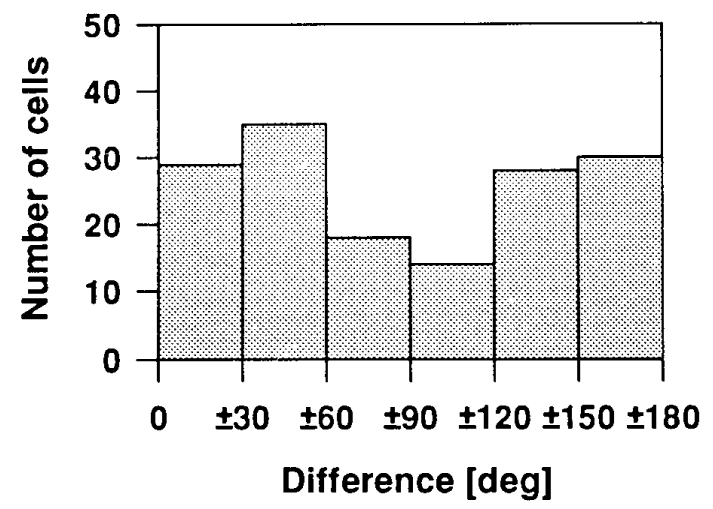

FIG. 14. Correlation of the preferred direction of pursuit and the direction of the gradient of the regression plane in area MST. For details of computation see text. Angular differences are shown as a circular scatter plot $(A)$ and as a histogram $(B)$. Statistical analysis did not reveal any correlation between both directions. 


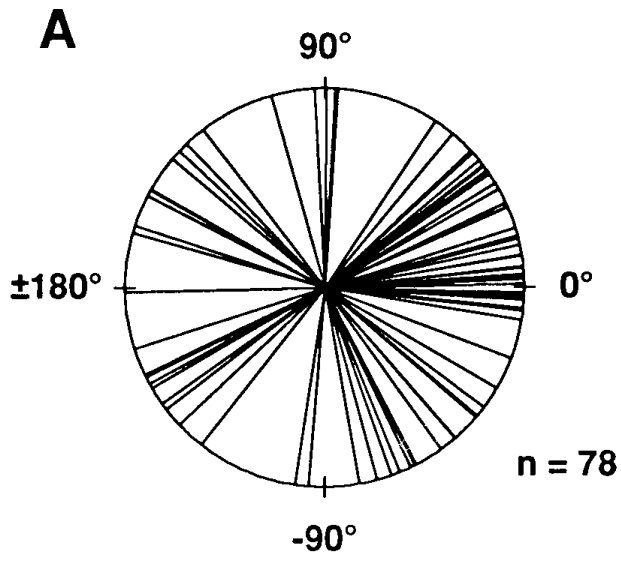

B

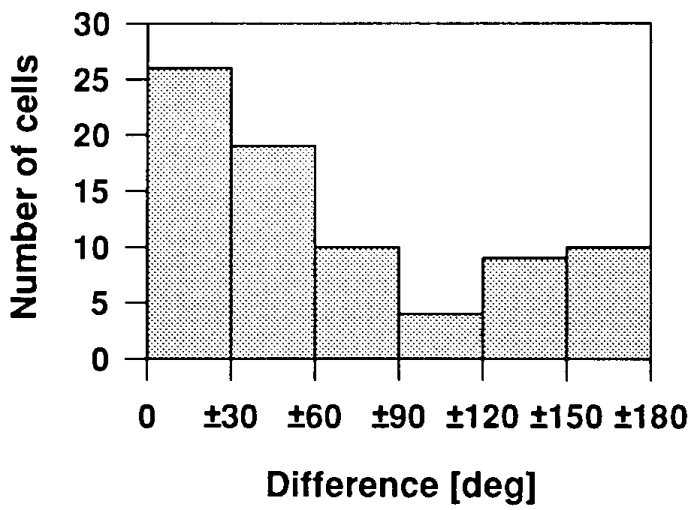

FIG. 15. Correlation of the directions of the gradient of the regression planes fit to the responses of MST neurons tested in the fixation and the pursuit paradigm. In the circular scatter plot $(A)$, each line represents the angular difference between both gradient directions for a single neuron. The histogram $(B)$ depicts the distribution of the absolute values of the angular differences. For details of computation see text. For $70 \%$ of the investigated MST neurons the angular difference between both gradients directions was less than $\pm 90^{\circ}$.

spheres. Thus all $D_{\text {RF-Ecc }}$ values ranged from $90^{\circ}$ to $270^{\circ}$. Neurons with zero eccentricity had to be excluded from this analysis. As can also be seen in Fig. 16, $D_{\text {Grad }}$ was not correlated with $D_{\text {RF-Ecc }}$, either for area MT or for area MST. The correlation coefficients of the computed linear regressions (least-square method) in Fig. 16, $A-F$, were all smaller than $r^{2}=0.015$.

A total of 168 neurons was tested in the pursuit paradigm: 14 MT neurons as well as 154 MST neurons. A regression plane was fit to the response of each individual neuron. This approximation was significant at $P<0.05$ for $57 \%$ ( 8 of 14 ) of the MT neurons and $43.5 \%$ (67 of 154) of the MST neurons. For another $7.8 \%$ (12 of 154) of the MST neurons the approximation was nearly significant at $P<0.1$. The distribution of the slopes of these planes for area MST is shown in Fig. 17. Each data point in the central two-dimensional graph depicts the gradient of one individual plane. Statistical analysis ( $\chi^{2}$ test) did not reveal any deviation of the distribution of the direction of gradients from a uniform distribution $(P>0.2)$. Gaussian distributions as represented by the dotted lines within each histogram plot appeared to be the best approximation for the distributions of the slopes along the horizontal and vertical axis when compared with other statistical distributions.

Computing the mean response of the whole ensemble of neurons recorded led to a similar result as for the fixation paradigm. Mean relative activities (raw activity minus background activity) for the different starting positions were not significantly different from each other (analysis of variance: $P>0.5$ for area MT and $P>0.6$ for area MST), indicating nearly no effect of the position of the eyes in the orbit on the pursuit-related response at the population level. Figure $18 \mathrm{~A}$ shows a histogram indicating the mean relative activity plus SDs in area MST for the different starting locations. Figure $18 B$ shows the mean discharge plane for the relative discharges in area MST, obtained from averaging the approximated regression planes.

\section{IS C U S S I ON}

The goal of this study was to investigate whether a modulatory effect of eye position on the mean neuronal discharge during fixation, visual stimulation, or smooth pursuit can be shown already for neurons as early as in areas MT and MST in the STS of primates.

\section{Fixation paradigm}

For the majority of neurons tested in areas MT and MST the strength of stimulus-driven activity was affected by eye position. Preferred directions, however, proved to be unaffected by the position of the eyes in the orbit. This finding is in accordance with results for parietal areas LIP and 7A from Andersen et al. (1990b), who could show that the direction tuning of light-sensitive, memory, and saccade discharges of neurons from these areas was unaffected whereas the magnitude of the response was modulated by eye position.

The neuron's preferred stimulus directions and the direction of the gradient of the regression plane did not correlate with each other, indicating that these two response characteristics are used by the system in an independent manner. To localize a point in space one could have imagined a very simple stimulus-eye position encoding scheme where, e.g., the discharge for a rightward stimulus movement is increased for most rightward and decreased for most leftward eye positions. However, such an orderly covariation between preferred stimulus direction and direction of the gradient of the regression plane could not be found for the tested neurons.

Furthermore, the influence of the position of the eyes in the orbit proved to be independent from RF characteristics like size and eccentricity.

\section{Pursuit paradigm}

For $\sim 80 \%$ of the neurons studied in both areas MT and MST, activity during pursuit was modulated by eye position. In addition, modulatory effects turned out to be planar for most of the neurons, i.e., sampled neuronal discharges as a function of horizontal and vertical eye position could be approximated by a two-dimensional linear regression function. The proportion of affected STS neurons tested in the pursuit paradigm nearly coincides with the amount of affected neurons in areas LIP and 7A tested in a saccade task 


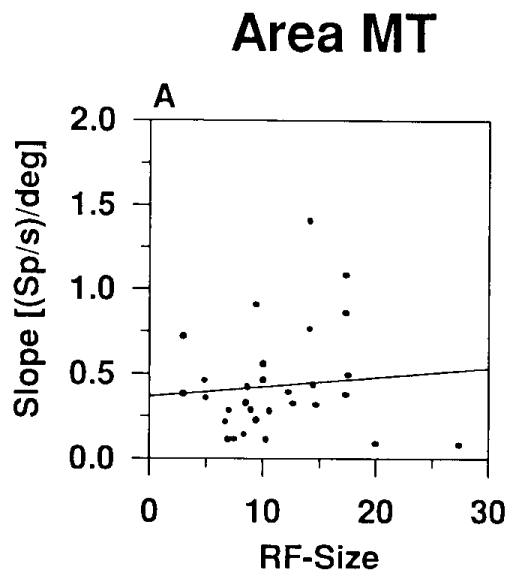

Area MST
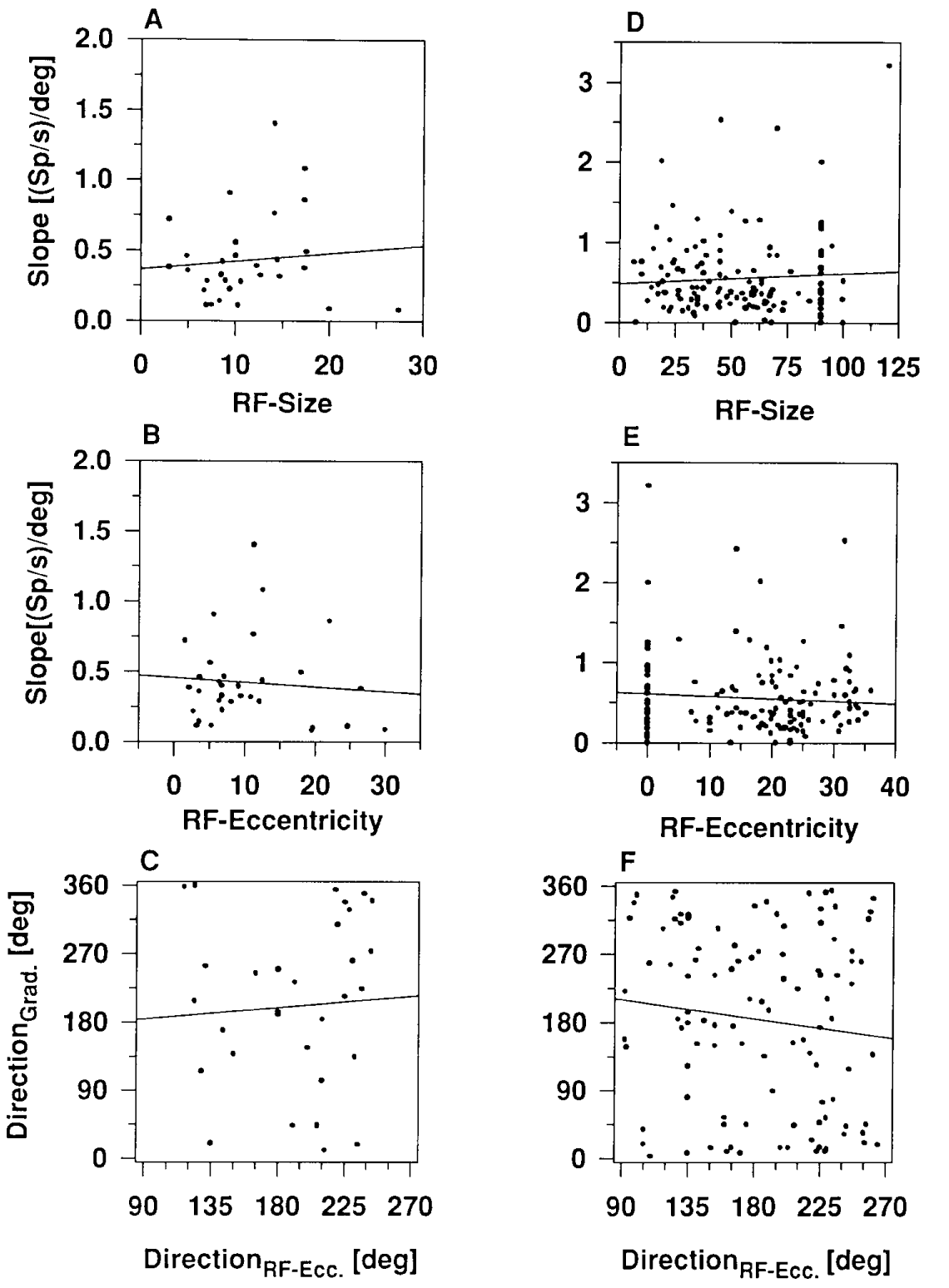

FIG. 16. Relationship between the eye position effect and RF size and RF location. $A-C$ : results for area MT. $D-F$ : results for area MST. RF size is defined as square root of the RF area. RF eccentricity is the distance of the geometric center of the RF from the fixation point, located usually at the screen center. Many MST neurons had RFs as large as the tangent screen $\left(90^{\circ} \times 90^{\circ}\right)$, causing the accumulation of data points at RF size 90 and eccentricity zero. Some very large MST RFs were mapped while the monkey fixated different locations on the screen. Some of these neurons proved to have RF sizes even larger than $90^{\circ} \times 90^{\circ}$. For computation of the direction of RF eccentricity and the direction of the gradient of the regression planes see text. The linear regression lines within each graph were derived employing the method of least squares. The eye position effect was clearly not correlated with $\mathrm{RF}$ size or RF eccentricity, either in area MT or in area MST.
(Andersen et al. 1990b). Also, the planar behavior of the influence of the position of the eyes in the orbit on the pursuit-related response resembles the characteristics found for saccade-related and light-sensitive discharge in areas LIP and $7 \mathrm{~A}$, where $80 \%$ of the tested neurons had a so-called "planar" or at least a "planar component" gain field (see also the accompanying paper for the influence of eye position on the activity during fixation in darkness and during smooth pursuit in areas LIP and 7A).

\section{Modulatory effect of eye position in different experimental paradigms}

The preferred directions of pursuit and the directions of the gradients of the regression planes were not correlated with each other. This coincides with our findings for response characteristics of neurons tested in the fixation paradigm. This finding does not confirm the prediction of a model from Goodman and Andersen (1989) for oculomotor (saccade)-related responses of eye-position-affected neurons (LIP). This model predicts a colinearity of the preferred saccade direction of the modeled neurons with the direction of the steepest increase of their gain fields. On the other hand, our finding of a missing correlation between both directions is in accordance with findings of Hepp et al. (1993) and van Opstal et al. (1995) for saccade-related activity in monkey superior colliculus. These authors could show in their studies that the modulatory influence of the position of the eyes in the orbit was not significantly related with the ON direction of saccades.

The same STS neurons tested in the pursuit paradigm often also showed a modulation of their activity when tested in the fixation paradigm. Eighty-six neurons were tested in both paradigms. For $\sim 70 \%$ of them $(75 \%$ in area MT and $70 \%$ in area MST) the directions of best responses (gradients of the regression planes) were directed into the same hemi- 
field, i.e., their response functions did more or less coincide. This is in accordance with findings from Andersen and colleagues from their study on memory, saccade-related, and light-sensitive responses in areas LIP and 7A (Andersen et al. 1990b). In that study, a colinearity was found (of about the same proportion of coincidence as in our study) of the gain fields for the neurons tested in different experimental paradigms. The authors hypothesized that the same cellular mechanisms might influence the activity of the neurons in different behavioral circumstances (see also the accompanying paper for our findings in areas LIP and 7A, which support the findings of Andersen and colleagues).

\section{Eye position effects at the system level}

The eye position effects were fairly uniformly distributed. This was true for both areas MT and MST and for both experimental tasks, the fixation and the pursuit paradigm. Statistical analysis revealed no significant preference for any direction of the modulatory influence of eye position on the neuronal discharge. The values of the slopes of the regression planes along each single axis (horizontal or vertical) were normally distributed. This observation is not in accordance with findings of Galletti and Battaglini (1989), who reported a slight tendency for area V3A neurons to respond better on average for more contralateral fixation locations, i.e., an anisotropy in the distribution of the gradients of the regression planes toward the contralateral hemifield. However, it corresponds to results from studies of Andersen et al. (1990b) on parietal areas LIP and 7A and Galletti et al. (1995) on parietooccipital area (PO). Both studies reported a uniform distribution of either the direction of gain field gradients approximated to saccade-related activity (areas
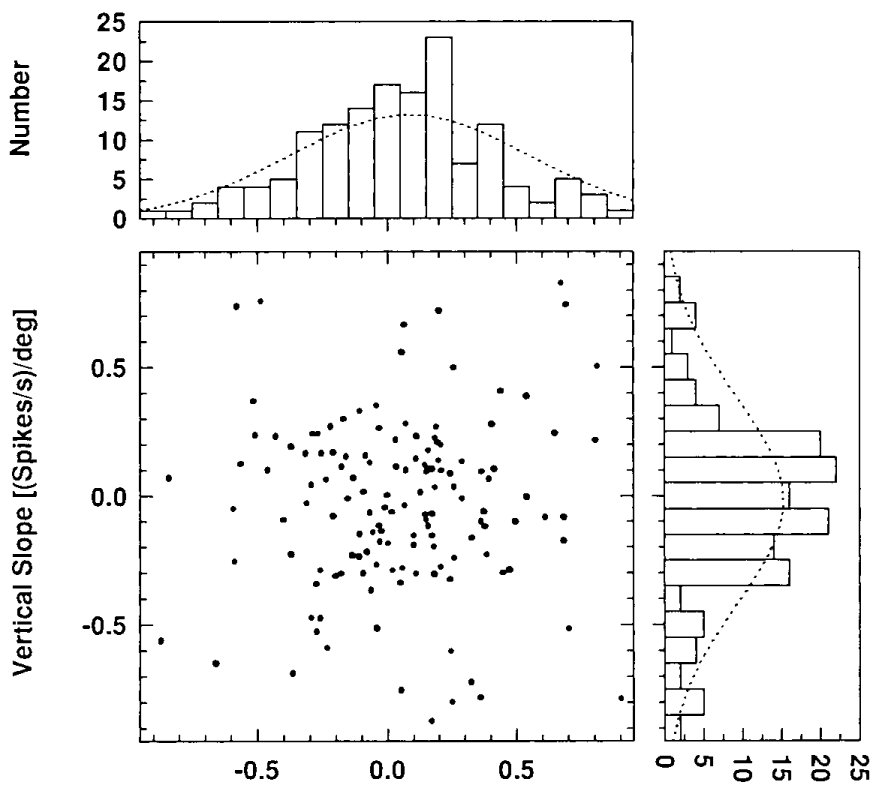

Horizontal Slope [(Spikes/s)/deg]

FIG. 17. Distribution of the gradients of the regression planes of area MST neurons tested in the pursuit paradigm. Middle: gradients of an individual linear regression plane are represented by a single data point. Statistical analysis proved the directions of the gradients to be uniformly distributed.

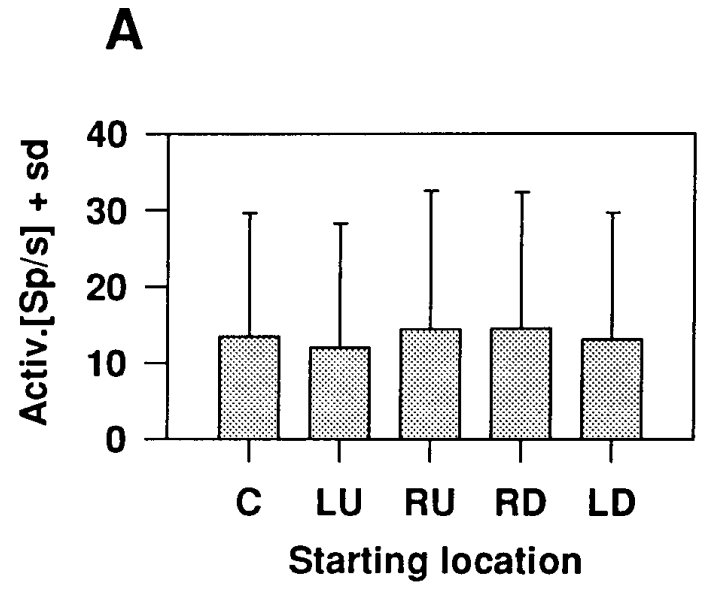

B

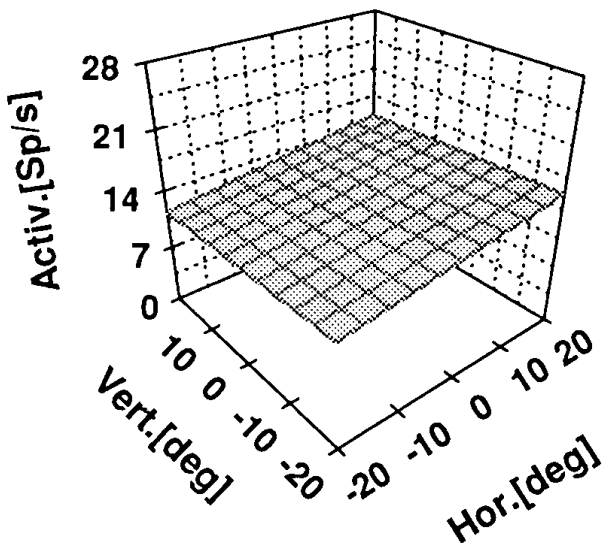

\section{$z[$ Spikes $/ \mathrm{s}]=0.086 \mathrm{x}-0.020 \mathrm{y}+13.57$$$
n=154
$$

FIG. 18. Population response for neurons from area MST tested in the pursuit paradigm. Histogram $(A)$ depicts the mean pursuit-related discharges (relative activity) plus SDs for the different starting locations of the pursuit target. Indication for the different starting location as in Fig. 13. The discharges for the different starting location were not significantly different, indicating that the modulatory effect of eye position is evenly distributed at the population level. The mean population response plane $(B)$ was obtained by averaging all linear regression planes. The resulting response plane turned out to be essentially flat.

LIP and 7A) or fixation point locations evoking peak activity $($ area $\mathrm{PO})$.

\section{Eye position effects and the hypothesis of a coordinate transformation}

The influence of the position of the eyes in the orbit seems to be quite a common phenomenon in the dorsal pathway of the monkey visual system. Including our study, the effect has been shown for areas V3A, V6, MT, MST, LIP, and 7A. It was suggested that these eye-position-dependent cells could be involved in the generation of an internal map of the external visual environment in which the object's position in space is encoded in a nonretinocentric frame of reference (Andersen et al. 1990b; Bremmer and Hoffmann 1995; Galletti and Battaglini 1989; Gnadt et al. 1991). These ideas were buttressed by findings from several theoretical studies 
on eye position effects (Andersen et al. 1993; Goodman and Andersen 1989, 1990; Mazzoni et al. 1991; Pouget et al. 1993). Most theoretical considerations thereby were more or less based on the influential study by Zipser and Andersen (1988). Briefly, Zipser and Andersen trained a three-layer back-propagation network to represent in the output layer space in a nonretinocentric frame of reference. By studying cell characteristics of the trained network, they found that the discharge characteristics of neurons in the hidden layer very much resembled the real discharge characteristics of neurons in parietal areas LIP and 7A. All mentioned hypotheses share the common idea that not a single neuron but the whole population of cells located within an area might be capable of transforming the visual signals into a head- or body-centered frame of reference. As theoretical considerations show (see, e.g., Salinas and Abbott 1994, 1995; Sanger 1994) a population coding requires, first, the activity of a considerable number of neurons with a broad tuning for a specific parameter. Second, the distribution of the tuning of these neurons has to be approximately uniform. Third, the average response has to be equal for the whole parameter space. As we have shown, most neurons in areas MT and MST are affected by the position of the eyes in the orbit. This tuning is quite broad and it can be shown mathematically ( $\mathrm{F}$. Bremmer, A. Pouget, and K.-P. Hoffman, unpublished data) that the planar tuning with respect to horizontal and vertical eye position is equivalent to a cosine tuning with respect to the direction of the gradient of the regression plane. The directions of the gradients of the regression planes were uniformly distributed. Thus neurons in areas MT and MST meet at least the first two criteria necessary for generating a population coding of eye position. We thus tested whether also the third criterion was met by the activity profiles of neurons in areas MT and MST. We computed the population responses for the cells tested in the pursuit and the fixation paradigm in both areas. It turned out that the eye position effect, found at the level of the single cell, was balanced out when all neuronal responses were taken together. The cumulative regression planes, resulting when averaging all neuronal responses, were essentially flat. Thus neurons in areas MT and MST meet all necessary criteria for a population coding of eye position. This might be taken as a hint for an ongoing coordinate transformation of the incoming sensory signals in areas MT and MST.

\section{Different concepts of space representation in monkey cortex}

The hypothesis of a distributed network of neurons generating a nonretinocentric representation of the visual environment is not the only theory concerning spatial information processing in primate cortex. Another hypothesis emphasizes the functional significance of neurons, revealing visual RFs that remain spatially constant regardless of eye position. Galletti et al. (1993) could show this response characteristic for neurons in monkey superior parietal area V6. Fogassi et al. (1992) have found cells with similar response characteristics in monkey premotor cortex. This kind of neurons thus could provide at the single-cell level what at least theoretically could be retrieved from the whole population of cells influenced by eye position: a coding of stimulus location in a nonretinocentric frame of reference. Because also in these experiments monkeys were head fixed, it cannot be concluded whether these cells code in a head-, body-, or spacecentered frame of reference. The finding of spatiocentric encoding neurons, however, does not rule out the functional significance of retinocentric neurons influenced by eye position. Galletti et al. $(1993,1995)$ could find for the very same area V6 many neurons with a remarkable influence of eye position onto their discharge. This majority of neurons revealed retinocentric and not spatiocentric RF characteristics. It thus might be that specific projections from eye-positiondependent neurons in hierarchically lower dorsal stream areas or even functional circuits within area V6 lead to the responses of the spatiotopic neurons. The same could hold true for the monkey premotor area. Whereas Fogassi et al. (1992) could show the existence of neurons with spatially constant visual RFs, Boussaoud et al. (1993) revealed an influence of eye position on apparent visual neuronal responses in ventral and dorsal premotor cortex. Single neurons encoding in a nonretinocentric frame of reference thus could obtain their response characteristic by local circuits within area PM or by specific projections from visual dorsal stream areas.

We thank Drs. W. Werner and E. Bauswein for surgery on the second monkey, and H. Korbmacher for skillful technical assistance.

This work was supported by ESPRIT INSIGHT II, and by stipends to F. Bremmer from the Friedrich Ebert Stiftung and the Deutsche Forschungsgemeinschaft (KOGNET).

Address reprint requests to K.-P. Hoffmann.

Received 17 July 1995; accepted in final form 10 May 1996.

\section{REFERENCES}

AgGleton, J. P. AND Mishkin, M. Visual impairments in macaques following inferior temporal lesions are exacerbated selectively by additional damage to superior temporal sulcus. Behav. Brain Res. 39: 262-274, 1990.

ANDERSEN, R. A. Inferior parietal lobule functions in spatial perception and visuomotor integration. In: Handbook of Physiology. The Nervous System. Higher Functions of the Brain. Bethesda, MD: Am. Physiol. Soc., 1987, sect. 1, vol. V, p. 483-518.

ANDERSEN, R. A. Visual and eye movement functions of the posterior parietal cortex. Annu. Rev. Neurosci. 12: 377-403, 1989.

Andersen, R. A., Asanuma, C., Essick, G., And Siegel, R. M. Corticocortical connections of anatomically and physiologically defined subdivisions within the inferior parietal lobule. J. Comp. Neurol. 296: 65-113, 1990a.

Andersen, R. A., Bracewell, R. M., Barash, S., Gnadt, J. W., And FoGASSI, L. Eye position effects on visual, memory, and saccade-related activity in areas LIP and 7A of macaque. J. Neurosci. 10: 1176-1196, 1990 b.

Andersen, R. A., Essick, G. K., And Siegel, R. M. Encoding of spatial location by posterior parietal neurons. Science Wash. DC 230: 456-458, 1985.

Andersen, R. A., Snyder, L. H., Li, C.-S., And Stricanne, B. Coordinate transformation in the representation of spatial information. Curr. Opin. Neurobiol. 3: 171-176, 1993.

ANDERSEN, R. A. AND ZIPSER, D. The role of the posterior parietal cortex in coordinate transformations for visual-motor integration. Can. J. Physiol. Pharmacol. 66: 488-501, 1988.

Bettges, I. Untersuchungen über Petrinetze als Hilfsmittel zur Modellierung neurobiologischer Prozesse (PhD thesis). Bochum, Germany: RuhrUniv. Bochum, 1992.

Boussaoud, D., BARTh, T. M., AND Wise, S. P. Effects of gaze on apparent visual responses of monkey frontral cortex neurons. Exp. Brain Res. 91: 202-211, 1993.

Boussaoud, D., Ungerleider, L. G., and Desimone, R. Pathways for motion analysis: cortical connections of the medial superior temporal and 
fundus of the superior temporal visual areas in the macaque. J. Comp. Neurol. 296: 462-495, 1990.

Braun, D., Weber, H., Mergner, T., and Schulte-Mönting, J. Saccadic reaction times in patients with frontal and parietal lesions. Brain 115: 1359-1386, 1992.

Bremmer, F. AND HofFmann, K.-P. Eye-position effects on pursuit related responses in area LIP of macaque monkey. In: Eye Movement Research: Mechanisms, Processes and Applications, edited by J. Findlay, R. Walker, and R. W. Kentridge. Amsterdam: Elsevier, 1995, p. 225-236.

Celebrini, S. ANd Newsome, W. T. Neuronal and psychophysical sensitivity to motion signals in extrastriate area MST of the macaque monkey. J. Neurosci. 14: 4109-4124, 1994.

Collewijn, H. AND TAMminga, E. P. Human fixation and pursuit in normal and open-loop conditions: effects of central and peripheral retinal targets. J. Physiol. Lond. 379: 109-129, 1986.

Duffy, C. J. AND WuRTZ, R. H. Sensitivity of MST neurons to optic flow stimuli. I. A continuum of response selectivity to large-field stimuli. $J$. Neurophysiol. 65: 1329-1345, 1991a.

DuFFY, C. J. AND WuRTZ, R. H. Sensitivity of MST neurons to optic flow stimuli. II. Mechanisms of response selectivity revealed by small-field stimuli. J. Neurophysiol. 65: 1346-1359, 1991b.

DuFFY, C. J. AND WURTZ, R. H. Response of monkey MST neurons to optic flow stimuli with shifted centers of motion. J. Neurosci. 15: 5192-5208, 1995.

Felleman, D. J. AND van Essen, D. C. Distributed hierarchical processing in the primate cerebral cortex. Cereb. Cortex 1: 1-47, 1991.

Fogassi, L., Gallese, V., Di Pellegrino, G., Fadiga, L., Gentilucci, M., Luppino, G., Matelli, M., Pedotti, A., and Rizzolatti, G. Space coding by premotor cortex. Exp. Brain Res. 89: 686-690, 1992.

Galletti, C. AND BATTAGLinI, P. P. Gaze-dependent visual neurons in area V3A of monkey prestriate cortex. J. Neurosci. 9: 1112-1125, 1989.

Galletti, C., Battaglini, P. P., And Fattori, P. Functional properties of neurons in the anterior bank of the parieto-occipital sulcus of the macaque monkey. Eur. J. Neurosci. 3: 452-461, 1991.

Galletti, C., Battaglini, P. P., And Fattori, P. Parietal neurons encoding spatial locations in craniotopic coordinates. Exp. Brain Res. 96: $221-$ 229, 1993.

Galletti, C., Battaglini, P. P., and Fattori, P. Eye position influence on the parieto-occipital area PO (V6) of the macaque monkey. Eur. J. Neurosci. 7: 2486-2501, 1995.

Gnadt, J. W., Bracewell, R. M., and Andersen, R. A. Sensorimotor transformations during eye movements to remembered visual targets. Vision Res. 31: 693-715, 1991

Goodale, M. A. AND MilnER, A. D. Separate visual pathways for perception and action. Trends Neurosci. 15: 2025, 1992.

GoOdMAn, S. AND ANDERsEn, R. A. Microstimulations of a neural network model for visually guided saccades. J. Cognit. Neurosci. 1: 317-326, 1989.

Goodman, S. J. AND ANDERsEn, R. A. Algorithm programmed by a neural network model for coordinate transformation. Int. Joint Conf. Neural Networks 2: 381-386, 1990.

Hepp, K., Suzuki, Y., And Henn, V. The gain field gradients of short lead burst neurons are independent of saccadic on-directions. Soc. Neurosci. Abtsr. 19: 684, 1993.

Hoffmann, K.-P. AND DistLer, C. Quantitative analysis of visual recreptive fields of neurons in nucleus of the optic tract and dorsal terminal nucleus of the accessory tract in macaque monkey. J. Neurophysiol. 62: 416428, 1989.

ILG, U. J. And Hoffmann, K.-P. Functional grouping of the cortico-pretectal projection. J. Neurophysiol. 70: 867-869, 1993.

Judge, S. J., Richmond, B. J., AND Chu, F. C. Implantation of magnetic search coils for measurement of eye position: an improved method. Vision Res. 20: 535-538, 1980.

Karnath, H. O., Schenkel, P., And Fischer, B. Trunk orientation as the determining factor of the "contralateral" deficit in the neglect syndrome and as the physical anchor of the internal representation of body orientation in space. Brain 114: 1997-2014, 1991.

Komatsu, H. AND WuRTZ, R. H. Relation of cortical areas MT and MST to pursuit eye movements. I. localization and visual properties of neurons J. Neurophysiol. 60: 580-603, 1988.

LAPPE, M., Bremmer, F., AND Hoffmann, K.-P. How to use non-visual information for optic flow processing in monkey visual cortical area MSTd. In: ICANN 94, edited by M. Marinaro and P. G. Morasso. Berlin: Springer-Verlag, 1994, p. 46-49.

LAPPE, M. AND RAUSCHECKER, J. P. A neural network for the processing of optic flow from ego-motion in man and higher mammals. Neural Comput. 5: 374-391, 1993.

LAPPE, M. AND RauschecKer, J. P. Heading detection from optic flow. Nature Lond. 369: 712-713, 1994.

LYNCH, J. C. The functional organization of posterior parietal association cortex. Behav. Brain Sci. 3: 485-534, 1980.

LYNCH, J. C. Saccade initiation and latency deficits after combined lesions of the frontal and posterior eye fields in monkeys. J. Neurophysiol. 68: 1913-1916, 1992.

LyNCH, J. AND MCLAREN, J. W. Effects of posterior parietal lobe lesion on optokinetic nystagmus in rhesus monkey. Federation Proc. 38: 899, 1979.

Mazzoni, P., ANDERSEN, R. A., AND JoRdAn, M. I. A more biologically plausible learning rule for neural networks. Proc. Natl. Acad. Sci. USA 88: 4433-4437, 1991.

McLaRen, J. W. AND LyNCH, J. C. Quantitative studies of optokinetic nystagmus in monkeys before and after lesions of parieto-occipital association cortex. Soc. Neurosci. Abstr. 5: 797, 1979.

Morel, A. AND Bullier, J. Anatomical segregation of two cortical visual pathways in the macaque. Visual Neurosci. 4: 555-578, 1990.

Pouget, A., Fisher, S. A., AND SEJNOwsKi, T. J. Egocentric spatial representation in early vision. J. Cognit. Neurosci. 5: 150-161, 1993.

RASHBASS, C. The relationship between saccadic and smooth tracking eye movements. J. Physiol. Lond. 159: 326-338, 1961.

RolLs, E. T. Neural organization of higher visual functions. Curr. Opin. Neurobiol. 1: 274-278, 1991.

Salinas, E. And Aвbott, L. F. Vector reconstruction from firing rates. $J$. Comp. Neurosci. 1: 89-107, 1994.

Salinas, E. AND Aвbott, L. F. Transfer of coded information from sensory to motor networks. J. Neurosci. 15: 6461-6474, 1995.

SANGER, T. D. Theoretical considerations for the analysis of population coding in motor cortex. Neural Comput. 6: 29-37, 1994.

SchoppmanN, A. AND HoffMANN, K.-P. Continuous mapping of directional selectivity in the cat's visual cortex. Neurosci. Lett. 2: 177-181, 1976.

SteIN, J. F. Space and the parietal association areas. In: Brain and Space, edited by J. Paillard. Oxford, UK: Oxford Univ. Press, 1991, p. 185222.

STEIN, J. F. The representation of egocentric space in the posterior parietal cortex. Behav. Brain Sci. 15: 691-700, 1992.

Tanaka, K., Sugita, Y., Moriya, M., And Saito, H.-A. Analysis of object motion in the ventral part of the medial superior temporal area of the macaque visual cortex. J. Neurophysiol. 69: 128-142, 1993.

UnGERLEIDER, L. G. AND Desimone, R. Projections to the superior temporal sulcus from the central and periphereal field representations of V1 and V2. J. Comp. Neurol. 248: 147-163, 1986.

UngerLeIDER, L. G. AND MishKin, M. Two cortical visual systems. In: The Analysis of Visual Behavior, edited by D. J. Ingle, M. A. Goodale, and R. J. Mansfield. Cambridge, MA: MIT Press, 1982, p. 549-586.

van Essen, D. C. AND Maunsell, J. H. R. Two dimensional maps of the cerebral cortex. J. Comp. Neurol. 191: 255-281, 1980.

van Opstal, A. J., Hepp, K., Suzuki, Y., and Henn, V. Influence of eye position on activity in monkey superior colliculus. J. Neurophysiol. 74: $1593-1610,1995$.

WÖRGÖTTER, F. AND EYSEL, U. T. Quantitative determination of orientational and directional components in the response of visual cortical cells to moving stimuli. Biol. Cybern. 57: 349-355, 1987.

ZeKI, S. Anatomical guides to the functional organization of the visual cortex. In: Neurobiology of Neocortex, edited by P. Rakic and W. Singer. New York: Wiley, 1988, p. 241-251.

ZIPSER, D. AND ANDERSEN, R. A. A back-propagation programmed network that simulates response properties of a subset of posterior parietal neurons. Nature Lond. 331: 679-684, 1988. 